\title{
EXCEPTIONAL POINTS OF DEGENERACY IN TRAVELING WAVE TUBES
}

\author{
ALEXANDER FIGOTIN
}

\begin{abstract}
Traveling wave tube (TWT) is a powerful vacuum electronic device used to amplify radio-frequency $(\mathrm{RF})$ signals with numerous applications, including radar, television and telephone satellite communications. TWT design in a nutshell comprises of a pencil-like electron beam (ebeam) in vacuum interacting with guiding it slow-wave structure (SWS). In our studies here the e-beam is represented by one-dimensional electron flow and SWS is represented by a transmission line (TL). The interaction between the e-beam and the TL is modeled by an analytic theory that generalizes the well-known Pierce model by taking into account the so-called space-charge effects particularly electron-to-electron repulsion (debunching). Many important aspects of the analytic theory of TWTs have been already analyzed in our monograph on the subject. The focus of the studies here is on degeneracies of the TWT dispersion relations particularly on exceptional points of degeneracy and their applications. The term exceptional point of degeneracy (EPD) refers to the property of the relevant matrix to have nontrivial Jordan block structure. Using special parameterization particularly suited to chosen EPD we derive exact formulas for the relevant Jordan basis including the eigenvectors and the so-called root vector associated with the Jordan block. Based on these studies we develop constructive approach to sensing of small signals.
\end{abstract}

\section{INTRODUCTION}

There is growing interest to electromagnetic system exhibiting Jordan eigenvector degeneracy, which is a degeneracy of the system evolution matrix when not only some eigenvalues coincide but the corresponding eigenvectors coincide also. The degeneracy of this type is sometimes referred to as exceptional point of degeneracy (EPD), [Kato, II.1]. A particularly important class of applications of EPDs is sensing, CheN]. [PeLiXu, [Wie, [Wie1], [KNAC], OGC].

In our prior work in [FigSynbJ], FigSynbJ, FigPert we advanced and studied simple circuits exhibiting EPDs and their applications to sensing. Operation of electric circuits though is limited to frequencies up to hundreds of $\mathrm{MHz}$, and to overcome this limitation other physical systems that can operate at higher frequencies must be considered. Our prior studies of traveling wave tubes (TWT) in [FigTWTbk, 4, 7, 13, 14, 54, 55] demonstrate that TWTs always have EPDs. Operating frequencies of TWTs can go up to hundreds of $\mathrm{GHz}$ and even into $\mathrm{THz}$ frequency range, BoosVE], Burt and for this reason they are the primary subject of our studies here. For more applications of EPDs for traveling wave tubes see [OTC], OVFC], OVFC1], [VPFC].

We start with a concise review of the basics of traveling wave tubes. Traveling wave tube (TWT) utilizes the energy of the electron beam (e-beam) as a flow of free electrons in a vacuum and converts it into an RF signal, see Fig. 1. To facilitate energy conversion and signal amplification, the electron beam is enclosed in the so-called slow wave structure (SWS), which supports waves that are slow enough to effectively interact with the e-beam. As a result of this interaction, the kinetic energy of electrons is converted into the electromagnetic energy stored in the field, Gilm1], Tsim], [Nusi, 2.2], [SchaB, 4]. Consequently, the key operational principle of a TWT is a positive feedback interaction between the slow-wave structure and the flow of electrons. The physical mechanism of radiation generation and its amplification is the electron bunching caused by the acceleration and deceleration of electrons along the e-beam.

Key words and phrases. Traveling wave tube, TWT, exceptional point of degeneracy (EPD), Jordan block, perturbations, instability, sensitivity. 


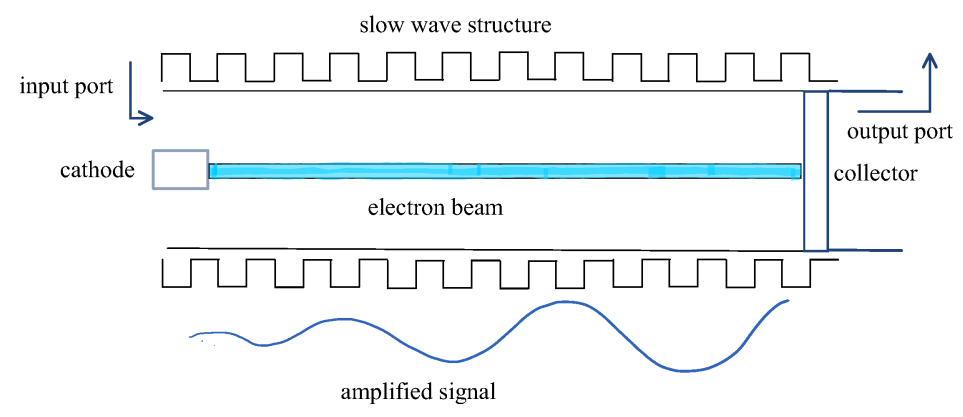

FiguRE 1. The upper picture is a schematic presentation of a traveling wave tube. The lower picture shows an RF perturbation in the form of a space-charge wave that gets amplified exponentially as it propagates through the traveling wave tube.

A schematic sketch of typical TWT is shown in Fig. 1. Such a typical TWT consists of a vacuum tube containing an e-beam that passes down the middle of an SWS such as an RF circuit. It operates as follows. The left end of the RF circuit is fed with a low-powered RF signal to be amplified. The SWS electromagnetic field acts upon the e-beam causing electron bunching and the formation of the so-called space-charge wave. In turn, the electromagnetic field generated by the space charge wave induces more current back into the RF circuit with a consequent enhancement of electron bunching. As a result, the EM field is amplified as the RF signal passes down the structure until a saturation regime is reached and a large RF signal is collected at the output. The role of the SWS is to provide slow-wave modes to match up with the velocity of the electrons in the e-beam. This velocity is usually a small fraction of the speed of light. Importantly, synchronism is required for effective in-phase interaction between the SWS and the e-beam with optimal extraction of the kinetic energy of the electrons. A typical simple SWS is the helix, which reduces the speed of propagation according to its pitch. The TWT is designed so that the RF signal travels along the tube at nearly the same speed as electrons in the e-beam to facilitate effective coupling. Technical details on the designs and operation of TWTs can be found in [Gilm1, [Nusi, 4] [PierTWT], [Tsim]. As for a rich and interesting history of traveling wave tubes, we refer the reader to [MAEAD] and references therein.

An effective mathematical model for a TWT interacting with the e-beam was introduced by Pierce [Pier51, I], PierTWT]. The Pierce model is one-dimensional; it accounts for the wave amplification, energy extraction from the e-beam and its conversion into microwave radiation in the TWT [Gilm1, Gilm, Nusi, 4], SchaB, 4], Tsim. This model captures remarkably well significant features of the wave amplification and the beam-wave energy transfer, and is still used for basic design estimates. In our paper [FigRey1], we have constructed a Lagrangian field theory by generalizing and extending the Pierce theory to the case of a possibly inhomogeneous MTL coupled to the e-beam. This work was extended to an analytic theory of multi-stream electron beams in traveling wave tubes in FigTWTbk.

According to our analytic theory the TWT dispersion relations always have EPDs which can be effectively found [FigTWTbk, 4, 7, 13, 14, 54, 55]. We study here the simplest TWT model that generalizes the Pierce model by integrating into it the space-charge effects. We introduce for this model a special parameterization that allow for (i) explicit representation of chosen EPD associated with a Jordan block of size 2; (ii) exact formulas for the Jordan basis including the eigenvectors and the so-called root vector associated with the Jordan block.

The paper is organized as follows. In Section 2 we review our analytic model of TWT introduced and studied in [FigTWTbk, 4, 24]. In Section 3 we carry out detailed studies of the relevant TWT 
matrix and its Jordan form. In Section 4 we (i) carry out the perturbation analysis of the relevant TWT matrix; (ii) develop constructive approach for using the TWT EPD for sensing of small signals.

While quoting monographs we identify the relevant sections as follows. Reference $[\mathrm{X}, \mathrm{Y}]$ refers to Section/Chapter "Y" of monograph (article) "X", whereas [X, p. Y] refers to page "Y" of monograph (article) "X". For instance, reference [2, VI.3] refers to monograph [2], Section VI.3; reference [2, p. 131] refers to page 131 of monograph [2].

\section{An ANAlytic model of the traVEling WAVE tube}

We concisely review here an analytic model of the traveling wave tube introduced and studied in our monograph [FigTWTbk, 4, 24]. According to this model an ideal TWT is represented by a single-stream e-beam interacting with single transmission line. This model is a generalization of the Pierce model [Pier51, I], [PierTWT] and its parameters are as follows. The main parameter describing the single-stream e-beam is e-beam coefficient

$$
\beta=\frac{\sigma_{\mathrm{B}}}{4 \pi} R_{\mathrm{sc}}^{2} \omega_{\mathrm{p}}^{2}=\frac{e^{2}}{m} R_{\mathrm{sc}}^{2} \sigma_{\mathrm{B}} \stackrel{\circ}{n}, \quad \omega_{\mathrm{p}}^{2}=\frac{4 \pi \grave{n} e^{2}}{m},
$$

where $-e$ is electron charge with $e>0, m$ is the electron mass, $\omega_{\mathrm{p}}$ is the e-beam plasma frequency, $\sigma_{\mathrm{B}}$ is the area of the cross-section of the e-beam, the constant $R_{\mathrm{sc}}$ is the plasma frequency reduction factor that accounts phenomenologically for finite dimensions of the e-beam cylinder as well as

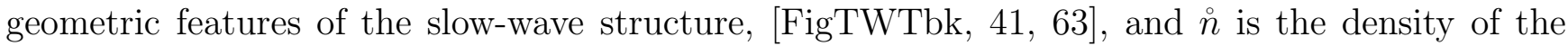
number of electrons. The single-stream e-beam has steady velocity $\stackrel{v}{v}>0$.

As for the single transmission line, its shunt capacitance per unit of length is a real number $C>0$ and its inductance per unit of length is another real number $L>0$. The coupling constant $0<b \leq 1$ is a number also, see [FigTWTbk, 3] for more details. The TL single characteristic velocity $w$ and the single TL principal coefficient $\theta$ defined by

$$
w=\frac{1}{\sqrt{C L}}, \quad \theta=\frac{b^{2}}{C} .
$$

Following to [FigTWTbk, 3] we assume that

$$
0<\stackrel{\circ}{0}<w
$$

2.1. TWT system Lagrangian and evolution equations. Following to developments in FigTWTbk we introduce the TWT principal parameter $\bar{\gamma}=\theta \beta$. This parameter in view of equations (2.1) and (2.2) can be represented as follows

$$
\gamma=\theta \beta=\frac{b^{2}}{C} \frac{\sigma_{\mathrm{B}}}{4 \pi} R_{\mathrm{sc}}^{2} \omega_{\mathrm{p}}^{2}=\frac{b^{2}}{C} \frac{e^{2}}{m} R_{\mathrm{sc}}^{2} \sigma_{\mathrm{B}} \stackrel{\circ}{n}, \quad \theta=\frac{b^{2}}{C}, \quad \beta=\frac{e^{2}}{m} R_{\mathrm{sc}}^{2} \sigma_{\mathrm{B}} \stackrel{\circ}{n} .
$$

The TWT-system Lagrangian $\mathcal{L}_{\mathrm{TB}}$ is defined by [FigTWTbk, 4, 24]

$$
\begin{gathered}
\mathcal{L}_{\mathrm{TB}}=\mathcal{L}_{\mathrm{Tb}}+\mathcal{L}_{\mathrm{B}} \\
\mathcal{L}_{\mathrm{Tb}}=\frac{L}{2}\left(\partial_{t} Q\right)^{2}-\frac{1}{2 C}\left(\partial_{z} Q+b \partial_{z} q\right)^{2}, \mathcal{L}_{\mathrm{B}}=\frac{1}{2 \beta}\left(\partial_{t} q+\stackrel{\circ}{*} \partial_{z} q\right)^{2}-\frac{2 \pi}{\sigma_{\mathrm{B}}} q^{2},
\end{gathered}
$$

where $q(z, t)$ and $Q(z, t)$ are charges associated with the e-beam and the TL defined as time integrals of the corresponding e-beam currents $i(z, t)$ and TL current $I(z, t)$, that is

$$
q(z, t)=\int^{t} i\left(z, t^{\prime}\right) \mathrm{d} t^{\prime}, \quad Q(z, t)=\int^{t} I\left(z, t^{\prime}\right) \mathrm{d} t^{\prime} .
$$


The corresponding Euler-Lagrange equations are the following system of second-order differential equations

$$
\begin{aligned}
L \partial_{t}^{2} Q-\partial_{z}\left[C^{-1}\left(\partial_{z} Q+b \partial_{z} q\right)\right] & =0, \\
\frac{1}{\beta}\left(\partial_{t}+\stackrel{v}{v} \partial_{z}\right)^{2} q+\frac{4 \pi}{\sigma_{\mathrm{B}}} q-b \partial_{z}\left[C^{-1}\left(\partial_{z} Q+b \partial_{z} q\right)\right] & =0, \quad \beta=\frac{\sigma_{\mathrm{B}}}{4 \pi} R_{\mathrm{sc}}^{2} \omega_{\mathrm{p}}^{2} .
\end{aligned}
$$

The Fourier transformation (see Section $\mathrm{A}$ ) in time $t$ and space variable $z$ of equations (2.7) and (2.8) yields

$$
\begin{gathered}
\left(k^{2} C^{-1}-\omega^{2} L\right) \hat{Q}+k^{2} C^{-1} b \hat{q}=0, \\
\frac{4 \pi}{\sigma_{\mathrm{B}}}\left[1-\frac{(\omega-\imath)^{2}}{R_{\mathrm{sc}}^{2} \omega_{\mathrm{p}}^{2}}\right] \hat{q}+k^{2} b C^{-1}[b \hat{q}+\hat{Q}]=0
\end{gathered}
$$

where $\omega$ and $k=k(\omega)$ are the frequency and the wavenumber, respectively, and functions $\hat{Q}=$ $\hat{Q}(\omega, k)$ and $\hat{q}=\hat{q}(\omega, k)$ are the Fourier transforms of the system vector variables $Q(t, z)$ and $q(t, z)$, see Appendix $\mathrm{A}$. In this case, the general TWT-system eigenmodes are of the form

$$
Q(z, t)=\hat{Q}(k, \omega) \mathrm{e}^{-\mathrm{i}(\omega t-k z)}, \quad q(z, t)=\hat{q}(k, \omega) \mathrm{e}^{-\mathrm{i}(\omega t-k z)},
$$

where as it turns out

$$
\hat{q}(k, \omega)=a_{0} \frac{1}{(u-\vartheta)^{2}}, \quad \hat{Q}(k, \omega)=a_{0} \frac{b w^{2}}{(u-\vartheta)^{2}\left(u^{2}-w^{2}\right)}, \quad u=\frac{\omega}{k} .
$$

In equation (2.12) $a_{0}$ is a constant of proper physical dimensions and, importantly, $u$ is the complexvalued characteristic velocity satisfying characteristic equation (2.18) provided below.

Notice that dividing equations (2.9) $-(2.10)$ by $k^{2}$ yields

$$
\begin{gathered}
\left(k^{2} C^{-1}-u^{2} L\right) \hat{Q}+C^{-1} b \hat{q}=0, \quad u=\frac{\omega}{k}, \\
\frac{4 \pi}{\sigma_{\mathrm{B}}}\left[\frac{u^{2}}{\omega^{2}}-\frac{(u-\hat{v})^{2}}{\Omega_{\mathrm{p}}^{2}}\right] \hat{q}+b C^{-1}[b \hat{q}+\hat{Q}]=0, \quad \Omega_{\mathrm{p}}=R_{\mathrm{sc}} \omega_{\mathrm{p}} .
\end{gathered}
$$

Concise matrix form of equations (2.13)-(2.14) is the following eigenvalue type problem for $k$ and $x$ assuming that $\omega$ is fixed

$$
M_{k \omega} x=0, \quad M_{k \omega}=\left[\begin{array}{rr}
-L \omega^{2}+\frac{k^{2}}{C} & \frac{b k^{2}}{C} \\
\frac{b k^{2}}{C} & \frac{b^{2} k^{2}}{C}+\frac{4 \pi}{\sigma}\left(1-\frac{(k \hat{v}-\omega)^{2}}{\Omega_{\mathrm{p}}^{2}}\right)
\end{array}\right], \quad x=\left[\begin{array}{c}
\hat{Q} \\
\hat{q}
\end{array}\right],
$$

The problem (2.15) is equivalent to another eigenvalue type problem for $u$ and $x$ assuming that $\omega$ is fixed

$$
\begin{gathered}
M_{u \omega} x=0, \quad M_{u \omega}=\left[\begin{array}{rr}
\frac{1}{u^{2}}-\frac{1}{w^{2}} & \\
\frac{b}{u^{2}} & \frac{b^{2}}{\gamma \breve{\omega}^{2}}+\frac{b}{u^{2}}-\frac{b^{2}(u-v)^{2}}{\gamma u^{2}}
\end{array}\right], \quad x=\left[\begin{array}{c}
\hat{Q} \\
\hat{q}
\end{array}\right], \\
u=\frac{\omega}{k}, \quad \breve{\omega}=\frac{\omega}{\Omega_{\mathrm{p}}} .
\end{gathered}
$$

where matrix $M_{u \omega}$ encodes the information about TWT eigenmodes and we refer to it as TWT principal matrix. As we show below this eigenvalue problem can be recast in terms of the theory of matrix polynomials reviewed in Section B. 
Notice the first equation in (2.16) is also kind of eigenvalue problem where being given $w, \stackrel{\circ}{v}, b$, $\gamma$ and $\breve{\omega}$ we need to find $u$ and two-dimensional nonzero vector $x$ that solve it. The problem of finding such $u$ is evidently reduced evidently to the characteristic equation

$$
\operatorname{det}\left\{M_{u \omega}\right\}=0 \text {, }
$$

where $M_{u \omega}$ is TWT principal matrix defined in equations (2.16). After elementary algebraic transformations of equation (2.17) we find that it is equivalent to the following equation

$$
\mathscr{D}(u, \gamma)=\frac{\gamma}{w^{2}-u^{2}}+\frac{(u-\stackrel{v}{2})^{2}}{u^{2}}=\frac{1}{\breve{\omega}^{2}}, \quad \breve{\omega}=\frac{\omega}{\Omega_{\mathrm{p}}}, \quad u=\frac{\omega}{k},
$$

and we refer to function $\mathscr{D}(u, \gamma)$ as the characteristic function. We refer to solutions of the characteristic equation (2.18) as characteristic velocities. Since the dimensionless frequency $\breve{\omega}$ in characteristic equation (2.18) is real, the characteristic equation is equivalent to the following system of equations:

$$
\Im\{\mathscr{D}(u, \bar{\gamma})\}=0, \quad \Re\{\mathscr{D}(u, \bar{\gamma})\}=\frac{1}{\breve{\omega}^{2}} \geq 0 .
$$

With the above equations in mind, we denote the set of all characteristic velocities $u$ by $\mathscr{U}_{\mathrm{TB}}^{+}=$ $\mathscr{U}_{\mathrm{TB}}^{+}(\bar{\gamma})$

$$
\mathscr{U}_{\mathrm{TB}}^{+}=\mathscr{U}_{\mathrm{TB}}^{+}(\bar{\gamma})=\{u: \Re\{\mathscr{D}(u, \bar{\gamma})\} \geq 0\}
$$

with superscript "+" being a reminder of $\Re\{\mathscr{D}(u, \bar{\gamma})\} \geq 0$.

The TWT characteristic function $\mathscr{D}(u, \bar{\gamma})$ defined by equation (2.9) can be represented in the form

$$
\mathscr{D}(u, \gamma)=\gamma \mathscr{D}_{\mathrm{T}}(u)+\mathscr{D}_{\mathrm{B}}(u)=\frac{\gamma}{w^{2}-u^{2}}+\frac{(u-\stackrel{\circ}{v})^{2}}{u^{2}}, \quad 0<\stackrel{\circ}{v}<w, \quad \gamma=\theta \beta,
$$

where $\mathscr{D}_{\mathrm{T}}(u)$ and $\mathscr{D}_{\mathrm{B}}(u)$ are respectively characteristic functions of the TL and e-beam defined by

$$
\mathscr{D}_{\mathrm{T}}(u)=u^{-2} \Delta_{\mathrm{T}}(u)=\frac{1}{w^{2}-u^{2}}, \quad \mathscr{D}_{\mathrm{B}}(u)=u^{-2} \Delta_{\mathrm{B}}^{-1}(u)=\frac{(u-\stackrel{\circ}{v})^{2}}{u^{2}} .
$$

Functions $\Delta_{\mathrm{T}}(u)$ and $\Delta_{\mathrm{B}}(u)$ are defined in turn by

$$
\Delta_{\mathrm{T}}(u)=\frac{u^{2}}{w^{2}-u^{2}}, \quad \Delta_{\mathrm{B}}(u)=\frac{1}{(u-\dot{v})^{2}}, \quad u=\frac{\omega}{k} .
$$

Using dimensionless variables

$$
\breve{\omega}=\frac{\omega}{\Omega_{\mathrm{p}}}, \quad \Omega_{\mathrm{p}}=R_{\mathrm{sc}} \omega_{\mathrm{p}}, \quad \check{\gamma}=\frac{\gamma}{\dot{v}^{2}}, \quad \check{u}=\frac{u}{\dot{v}}=\frac{\omega}{k \dot{v}}, \quad \chi=\frac{w}{\dot{v}} .
$$

we can recast eigenmode equation (2.16) as follows

$$
M_{u \omega} x=0, \quad M_{u \omega}=\left[\begin{array}{rr}
\frac{1}{\check{u}^{2}}-\frac{1}{\chi^{2}} & \frac{b}{\check{u}^{2}} \\
\frac{b}{\check{u}^{2}} & \left(\frac{1}{\check{u}^{2}}+\frac{1}{\grave{\gamma}}\left(\frac{1}{\check{\omega}^{2}}-\frac{(\check{u}-1)^{2}}{\check{u}^{2}}\right)\right) b^{2}
\end{array}\right], \quad x=\left[\begin{array}{c}
\hat{Q} \\
\hat{q}
\end{array}\right] .
$$

It is convenient to recast the first equation into the standard matrix polynomial in $\check{u}$ by multiplying it by $\check{u}^{2}$. That action after elementary algebraic transformations yields

$$
\mathrm{M}_{u \omega} x=0, \quad \mathrm{M}_{u \omega}=D_{b}^{\prime}\left[\begin{array}{rr}
\check{u}^{2}-\chi^{2} & -\chi^{2} \\
\frac{\check{\gamma}}{\check{\omega}^{-2}-1} & \check{u}^{2}+\frac{2 \check{u}}{\check{\omega}^{-2}-1}+\frac{\check{\gamma}-1}{\check{\omega}^{-2}-1}
\end{array}\right] D_{b}, \quad x=\left[\begin{array}{c}
\hat{Q} \\
\hat{q}
\end{array}\right],
$$

where

$$
D_{b}^{\prime}=\left[\begin{array}{rr}
-\frac{1}{\chi^{2}} & 0 \\
0 & \frac{b\left(\check{\omega}^{-2}-1\right)}{\check{\gamma}}
\end{array}\right], \quad D_{b}=\left[\begin{array}{ll}
1 & 0 \\
0 & b
\end{array}\right]
$$


Matrix polynomial equation (2.26) can in turn be readily recast into the following equivalent form

$$
\mathrm{M}_{u \omega} x=0, \quad \mathrm{M}_{u \omega}=\left[\begin{array}{cc}
\check{u}^{2}-\chi^{2} & -\chi^{2} \\
\frac{\check{\gamma}}{\check{\omega}^{-2}-1} & \check{u}^{2}+\frac{2 \check{u}}{\check{\omega}^{-2}-1}+\frac{\check{\gamma}-1}{\check{\omega}^{-2}-1}
\end{array}\right], \quad x=\left[\begin{array}{c}
\hat{Q} \\
b \hat{q}
\end{array}\right] .
$$

We refer to matrix $\mathrm{M}_{u \omega}$ defined in equations (2.28) TWT matrix polynomial. The theory of matrix polynomials is reviewed in Section B. From now on the matrix monic polynomial in $\check{u}$ equation (2.28) will be our preferred form. The corresponding characteristic equation equivalent to $\operatorname{det}\left\{\mathrm{M}_{u \omega}\right\}=0$ and related to it characteristic function $\mathscr{D}(\check{u}, \check{\gamma})$ and the corresponding equation are as follows

$$
\mathscr{D}(\check{u}, \check{\gamma})=\frac{\check{\gamma}}{\chi^{2}-\check{u}^{2}}+\frac{(\check{u}-1)^{2}}{\check{u}^{2}}=\frac{1}{\breve{\omega}^{2}}
$$

The conventional plot of the characteristic function $\mathscr{D}(u, \gamma)$ helps to visualize solutions $u$ to the characteristic equations (2.18), that is, $\mathscr{D}(u, \bar{\gamma})=\frac{1}{\breve{\omega}^{2}}$, when the solutions are real-valued. But a non-real solution $u$ to the characteristic equation, that is when $\Im\{u\} \neq 0$ is "invisible" in the conventional plot of the characteristic function $\mathscr{D}(u, \bar{\gamma})$. To remedy this, we extend the conventional following to FigTWTbk, 8]. Suppose that for $D>0$ equation $\mathscr{D}(u, \bar{\gamma})=D$ has a complex-valued solution $u, \Im\{u\} \neq 0$. We then represent such complex $u$ in the plot of $\mathscr{D}(u, \bar{\gamma})$ as a point

$$
(\Re\{u\}, D) \text {, where } \mathscr{D}(u, \bar{\gamma})=D>0 .
$$

We distinguish such points $(\Re\{u\}, D)$ by plotting curves comprised from them as solid (brown). We refer to the so extended plot as the plot of the characteristic function with instability branches. We also refer to the endpoints $\left(\mathrm{u}_{0}, \mathscr{D}\left(\mathrm{u}_{0}, \bar{\gamma}\right)\right)$ of the instability branches as the characteristic function instability nodes. When generating plots and numerical examples we often use the following data

$$
\stackrel{\circ}{v}=1, \quad w=1.1, \quad \gamma=3 .
$$

Figures 2, 3, and 4 show plots of the characteristic function $\mathscr{D}(u, \gamma)$ defined in equations (2.18) with its instability branches.

2.2. Dispersion relations. Following to [FigTWTbk, 4] we define for any characteristic velocity $u$ from characteristic velocity set $\mathscr{U}_{\mathrm{TB}}^{+}(\bar{\gamma})$ as in equations (2.20) the TWT frequency function $\Omega(u)$ so that it is the frequency corresponding to $u$ in the characteristic equation (2.18), namely

$$
\Omega(u)=\frac{\Omega_{\mathrm{p}}}{\sqrt{\mathscr{D}(u, \bar{\gamma})}}>0, \quad \Omega_{\mathrm{p}}=R_{\mathrm{sc}} \omega_{\mathrm{p}}, \quad u \in \mathscr{U}_{\mathrm{TB}}^{+}(\bar{\gamma}), \quad \mathscr{D}(u, \bar{\gamma})>0 .
$$

Notice that by the definition of the characteristic velocity $u$, the value of $\mathscr{D}(u, \bar{\gamma})$ must be real and positive, and the square root $\sqrt{\mathscr{D}(u, \bar{\gamma})}$ is assumed to be positive as well. Consequently, $\Omega(u)$ is real-valued. Also note that if $u_{0}$ is a real-valued characteristic velocity, then for any real $u$ belonging to its sufficiently small vicinity, we have $\mathscr{D}(u, \bar{\gamma})>0$, implying that it is also a characteristic velocity. Consequently, $\Omega(u)$ defined by equation (2.32) is a real analytic function in the vicinity of $u_{0}$. We may also view the equation

$$
\Omega(u)=\frac{\Omega_{\mathrm{p}}}{\sqrt{\mathscr{D}(u, \bar{\gamma})}}=\omega
$$

as an equivalent form of the characteristic equation (2.18) for $u$ in the vicinity of $u_{0}$. Equation (2.33) can be viewed also as a form of the dispersion relations and refer to it velocity dispersion 


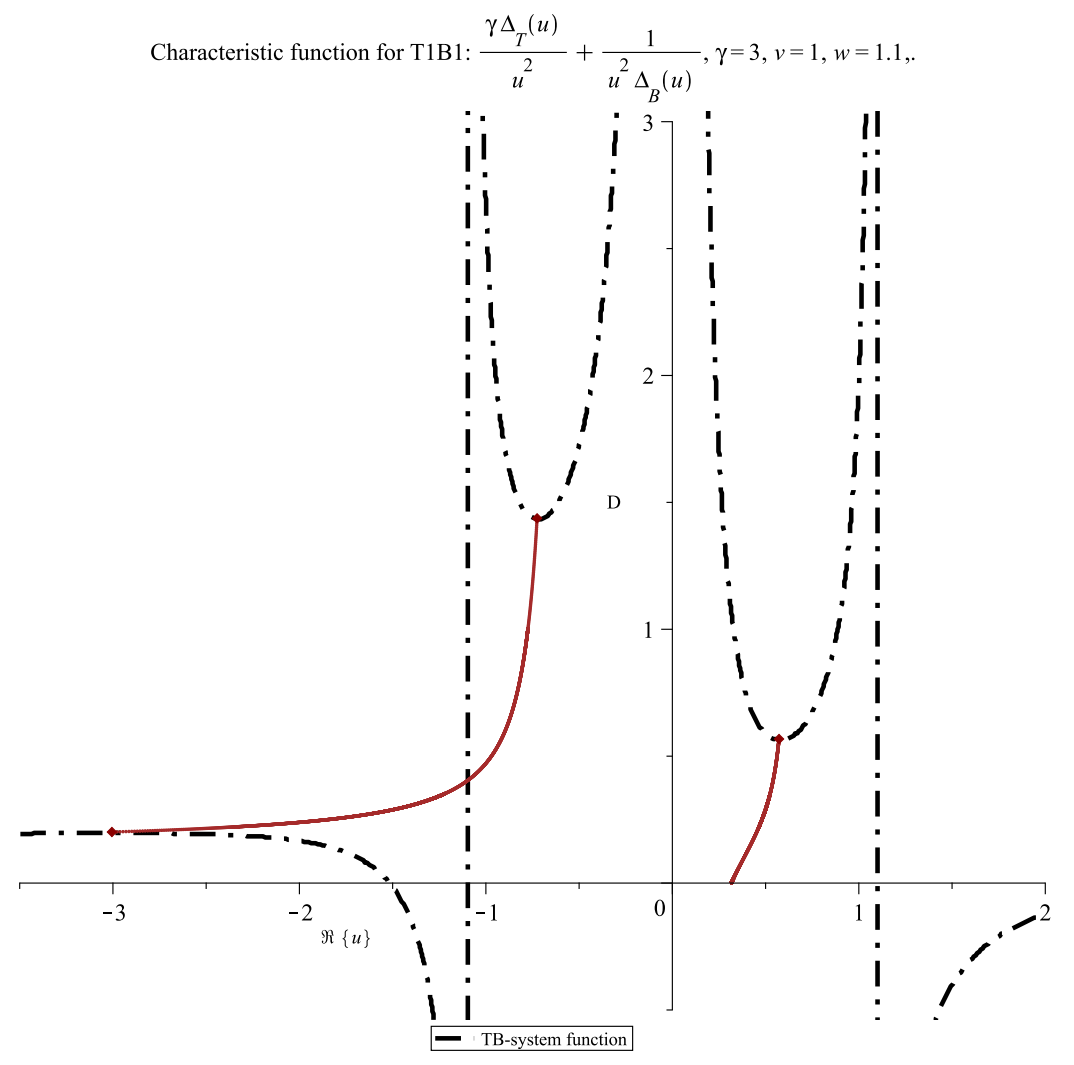

Figure 2. Single-stream e-beam coupled to a single TL. Plot of the characteristic function $\mathscr{D}(u, \gamma)=\frac{\gamma}{w^{2}-u^{2}}+\frac{(u-\grave{v})^{2}}{u^{2}}$ with its instability branches for $\stackrel{\circ}{v}=1, w=1.1$, $\gamma=3$ (horizontal axis $-\Re\{u\}$, vertical axis $-D$ ). The instability branches are represented by $\mathscr{D}(\Re\{u\}, \gamma)$, where $u$ is complex characteristic velocity, $\Im\{u\} \neq 0$, satisfying equations (2.9), that is, $\mathscr{D}(u, \gamma)=\frac{1}{\breve{\omega}^{2}}$. Dash-dot (black) lines represent the plot of $\mathscr{D}(u, \gamma)$ for real $u$, solid (brown) lines represent unstable branches with $\Im\{u\} \neq 0$. The characteristic function instability nodes are represented by solid (brown) diamond dots. Vertical dash-dot (black) straight lines represent asymptotes associated with real-valued poles of function $\gamma \mathscr{D}_{\mathrm{T}}(u)=\frac{\gamma}{w^{2}-u^{2}}$ and consequently of the characteristic function $\mathscr{D}(u, \gamma)$.

relation relation. Using the frequency function $\Omega(u)$, we naturally define the TWT wavenumber function $K(u)$ by the following equation:

$$
K(u)=\frac{\Omega(u)}{u}, \quad u \in \mathscr{U}_{\mathrm{TB}}^{+}(\bar{\gamma}), \quad \Omega(u)>0 .
$$

To visualize features of the TWT instability in its dispersion relation we proceed as follows FigTWTbk, 7]. We represent the set of all oscillatory and unstable modes of the TWT-system geometrically by the set $\Pi_{\mathrm{TB}}$ of the corresponding modal points $(k(\omega), \omega)$ and $(\Re\{k(\omega)\}, \omega)$ in the $k \omega$-plane. We name the set $\Pi_{\mathrm{TB}}$ as the dispersion-instability graph. To distinguish graphically points $(k(\omega), \omega)$ associated oscillatory modes when $k(\omega)$ is real-valued from points $(\Re\{k(\omega)\}, \omega)$ associated unstable modes when $k(\omega)$ is complex-valued with $\Im\{k(\omega)\} \neq 0$, we assign them colors as follows: (i) blue color is assigned to points $(k(\omega), \omega)$ when $k$ is real-valued; (ii) brown color is assigned to points $(\Re\{k(\omega)\}, \omega)$ when $k(\omega)$ is complex-valued with $\Im\{k(\omega)\} \neq 0$. We remind once again that every brown point $(\omega, \Re\{k(\omega)\})$ represents exactly two complex conjugate unstable modes associated with $\pm \Im\{k(\omega)\}$. We introduce then two subsets of the set $\Pi_{\mathrm{TB}}$, namely, $\Pi_{\mathrm{TB}}$ 


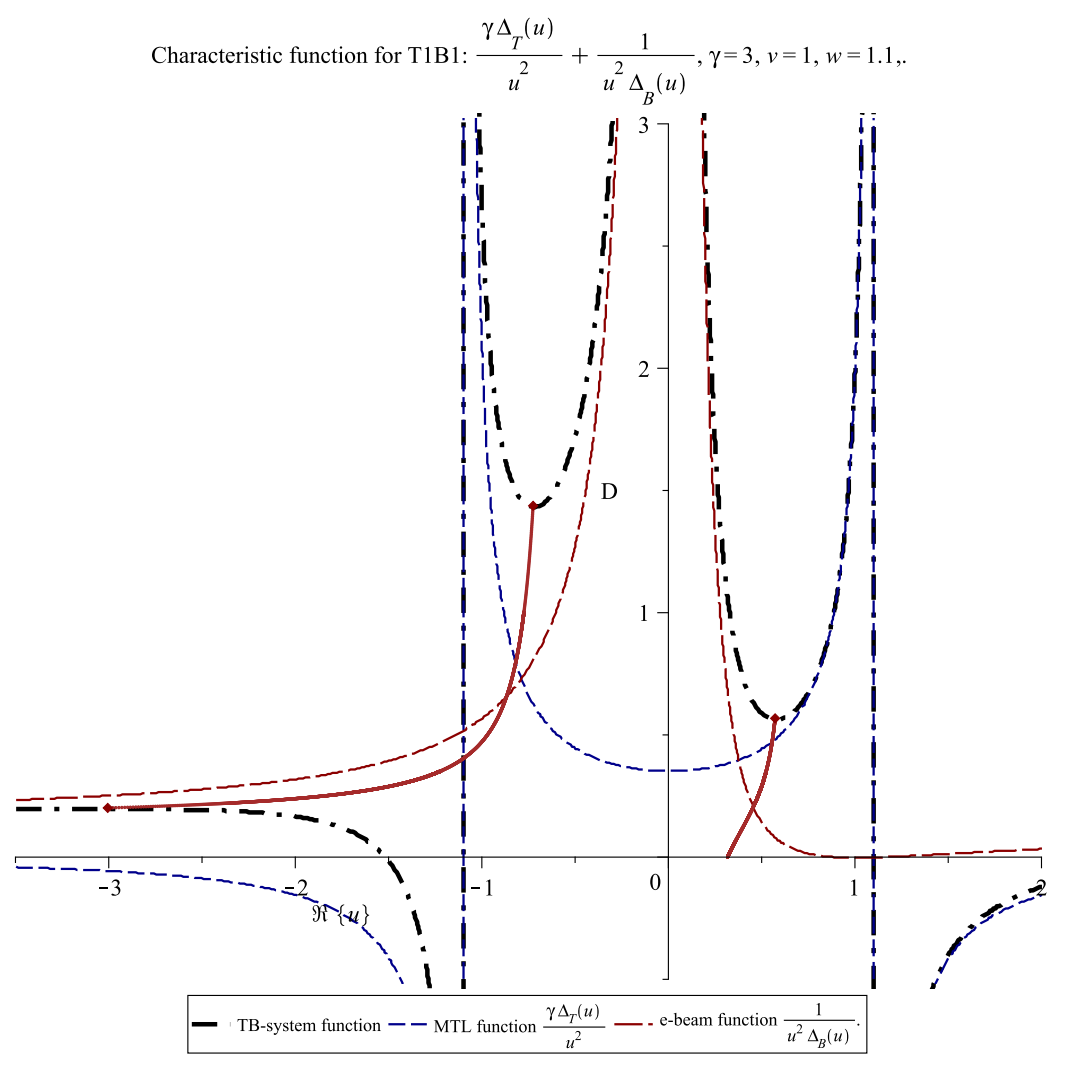

Figure 3. Single-stream e-beam coupled to a single TL. Plot of the characteristic function $\mathscr{D}(u, \gamma)=\frac{\gamma}{w^{2}-u^{2}}+\frac{(u-\grave{v})^{2}}{u^{2}}$ with its instability branches for $\stackrel{\circ}{v}=1, w=1.1$, $\gamma=3$ (horizontal axis $-\Re\{u\}$, vertical axis $-D$ ). The instability branches are represented by $\mathscr{D}(\Re\{u\}, \gamma)$, where $u$ is complex characteristic velocity, $\Im\{u\} \neq 0$, satisfying equations (2.9), that is, $\mathscr{D}(u, \gamma)=\frac{1}{\breve{\omega}^{2}}$. Dash-dot (black) lines represent the plot of $\mathscr{D}(u, \gamma)$ for real $u$, solid (brown) lines represent unstable branches with $\Im\{u\} \neq 0$, and dashed (brown, blue) lines represent, respectively, plots of functions $\gamma \mathscr{D}_{\mathrm{T}}(u)=\frac{\gamma}{w^{2}-u^{2}}$ and $\mathscr{D}_{\mathrm{B}}(u)=\frac{(u-\mathfrak{v})^{2}}{u^{2}}$. The characteristic function instability nodes are represented by solid (brown) diamond dots. Vertical dash-dot (black) straight lines represent asymptotes associated with real-valued poles of function $\frac{\gamma}{w^{2}-u^{2}}$ and consequently of the characteristic function $\mathscr{D}(u, \gamma)$.

and $\Pi_{\mathrm{TBu}}$ representing, respectively, all oscillatory and unstable modes. Evidently, the sets $\Pi_{\mathrm{TB}}$ and $\Pi_{\mathrm{TBu}}$ are disjoint, and they partition the set $\Pi_{\mathrm{TB}}$, that is, $\Pi_{\mathrm{TB}}=\Pi_{\mathrm{TBo}} \cup \Pi_{\mathrm{TBu}}$. According to the color assignments, the points of sets $\Pi_{\mathrm{TB}}$ and $\Pi_{\mathrm{TBu}}$ are, respectively, blue and brown. Fig. 5 shows a typical dispersion-instability graph for the data as in equation (2.31).

2.3. Nodal velocities, nodal function and equation. We consider here the nodal velocities which are the phase velocities that signify the onset of the TWT instability. According to our theory developed in FigTWTbk, 13, 30] the nodal velocities are solutions to the nodal equation

$$
\mathscr{R}(u)=-\frac{\partial_{u} \mathscr{D}_{\mathrm{B}}(u)}{\partial_{u} \mathscr{D}_{\mathrm{T}}(u)}=\frac{\stackrel{i}{v}(\dot{v}-u)\left(w^{2}-u^{2}\right)^{2}}{u^{4}}=\gamma,
$$

where functions $\mathscr{D}_{\mathrm{T}}(u)$ and $\mathscr{D}_{\mathrm{B}}(u)$ are defined by equations $(2.22)$ and $\gamma$ is TWT principal parameter defined equations (2.4). We refer to function $\mathscr{R}(u)$ in the nodal function. Figure 6 illustrates graphically features of the nodal function $\mathscr{R}(u)$ for a TWT composed of a single-stream e-beam 


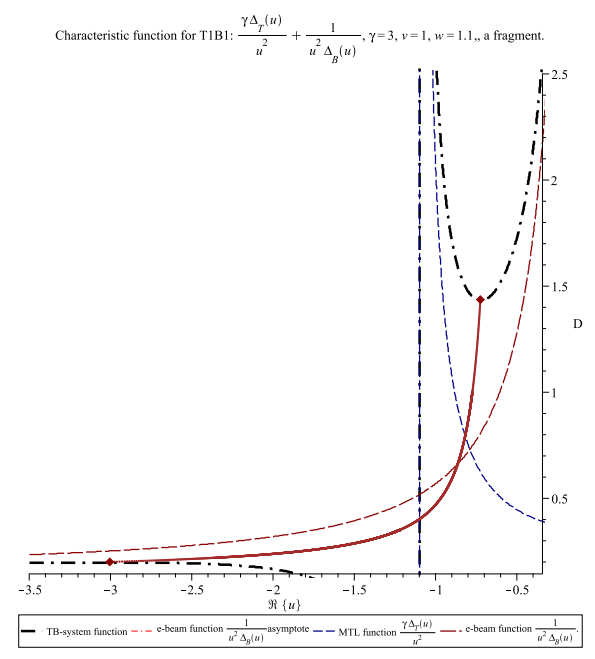

(a)

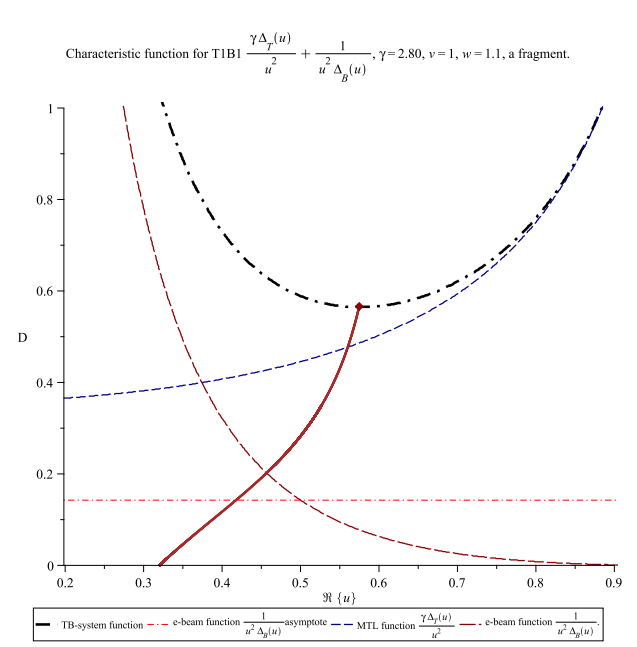

(b)

Figure 4. Single-stream e-beam coupled to a single TL. Zoomed fragments of the plot in Fig. 3 of the characteristic function $\mathscr{D}(\Re\{u\}, \gamma)$ with its instability branches (horizontal axis $-\Re\{u\}$, vertical axis $-D$ ) for $\stackrel{\circ}{v}=1, w=1.1, \gamma=3$ : (a) negative $u$ and $\Re\{u\}$; (b) positive $u$ and $\Re\{u\}$. The instability branches are represented by $\mathscr{D}(\Re\{u\}, \gamma)$, where $u$ is complex characteristic velocity, $\Im\{u\} \neq 0$, satisfying equations (2.9), that is, $\mathscr{D}(u, \gamma)=\frac{1}{\breve{\omega}^{2}}$. Dash-dot (black) lines represent the plot of $\mathscr{D}(u, \gamma)$ for real $u$, solid (brown) lines represent unstable branches with $\Im\{u\} \neq 0$, and dashed (brown, blue) lines represent plots of functions $\gamma \mathscr{D}_{\mathrm{T}}(u)=$ $\frac{\gamma}{w^{2}-u^{2}}$ and $\mathscr{D}_{\mathrm{B}}(u)=\frac{(u-v)^{2}}{u^{2}}$, respectively. The characteristic function instability nodes are represented by solid (brown) diamond dots. Vertical dash-dot (black) straight lines represent the asymptotes associated with real-valued poles of function $\frac{\gamma}{w^{2}-u^{2}}$ and consequently of the characteristic function $\mathscr{D}(u, \gamma)$.

and a single TL. Equation (2.35) can be recast in terms of dimensionless variables as follows:

$$
\mathscr{R}(\check{u})=\frac{(1-\check{u})\left(\chi^{2}-\check{u}^{2}\right)^{2}}{\check{u}^{4}}=\check{\gamma}, \quad \check{\gamma}=\frac{\gamma}{\dot{v}^{2}}, \quad \check{u}=\frac{u}{\grave{v}}, \quad \chi=\frac{w}{\grave{v}},
$$

where $\check{\gamma}$ is the dimensionless TWT principal parameter. If $u_{0}$ is the nodal velocity satisfying equation (2.36), then the corresponding instability node frequency $\omega_{0}$ as well as instability node wavenumber $k_{0}$ can be expressed in terms of the frequency function $\Omega(u)$ defined by equation (2.32), that is,

$$
\omega_{0}=\Omega\left(\mathrm{u}_{0}\right)=\frac{\Omega_{\mathrm{p}}}{\sqrt{\mathscr{D}\left(\mathrm{u}_{0}, \bar{\gamma}\right)}}, \quad k_{0}=\frac{\omega_{0}}{\mathrm{u}_{0}},
$$

where $\Omega(u)$ is defined by equations (2.32).

Observe that the nodal equation (2.35) has exactly three real-valued solutions: (i) one positive $\mathrm{u}^{+}(\bar{\gamma})>0$; (ii) two negative $\mathrm{u}_{1}^{-}(\bar{\gamma})<\mathrm{u}_{2}^{-}(\bar{\gamma})<0$. Notice also since in equation (2.32) $\gamma>0$ then $\mathbf{u}^{+}(\bar{\gamma})<1$ and hence we have

$$
0<\mathrm{u}^{+}(\bar{\gamma})<1
$$

2.4. Nodal points as points of degeneracy of the dispersion relations. The diamond (brown) dots Fig. [5identify the three points of the degeneracy of the dispersion relations. Vicinites of these points demonstrate graphically the transition to instability when points on dot-dashed 
Single-stream e-beam, single TL TWT. The dispersion relations for positive phase velocities. $\gamma=3, v=1, w=1.1$.

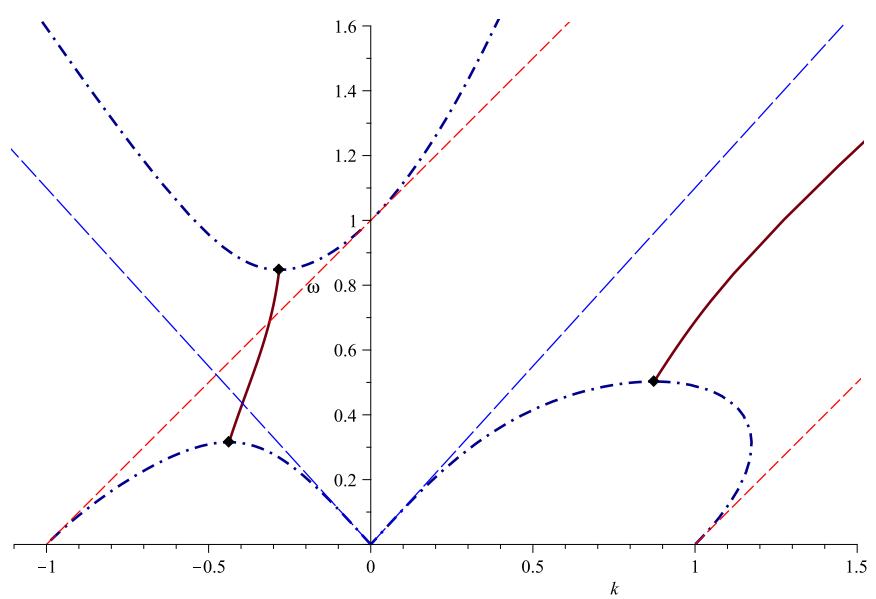

Figure 5. Single-stream e-beam coupled to a single TL. Dispersion-instability graph (horizontal axis $-\Re\{k\}$, vertical axis $-\omega$ ) for $\stackrel{\circ}{v}=1, w=1.1, \gamma=3$. Solid (brown) curves represent unstable branches with $\Im\{k\} \neq 0$, dash-dotted (black) lines represent oscillatory branches with $\Im\{k\}=0$, dashed lines represent the dispersion relations for uncoupled TL (blue, converging to the origin) and for an uncoupled e-beam (red). The instability nodes are represented by solid (brown) diamond dots.

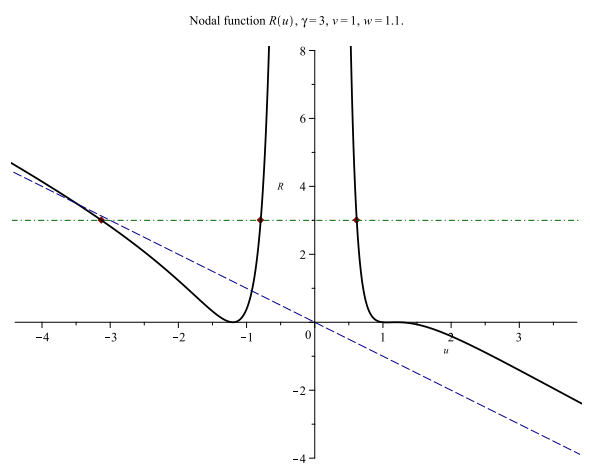

(a)

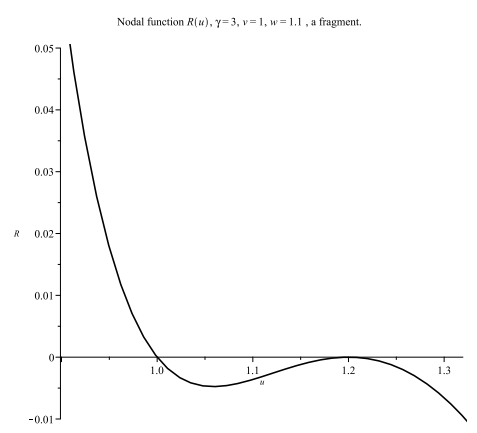

(b)

Figure 6. Single e-beam coupled to a single TL. Plot (a) of the nodal function $\mathscr{R}(u)=\frac{v(v-u)\left(w^{2}-u^{2}\right)^{2}}{u^{4}}$ and (b) its fragment (horizontal axis $-u$, vertical axis $-R$ ) for $\dot{v}=1, w=1.1$. Solid (black) line represents function $\mathscr{R}(u)$, and dashed (blue) line represents asymptotics function $\mathscr{R}(u)$ as $u \rightarrow \infty$, the dash-dot (green) line represents constant $\gamma=3$, and solid (brown) diamond dots represent solutions for the instability node equation (2.35), that is, $\mathscr{R}(u)=\gamma=3$.

(blue) lines associated with oscillatory modes with real phase velocities $u$ lines and real wavenumbers $k$ merge with points on solid (brown) lines associated with complex-conjugate pairs of unstable modes with non-real real phase velocities at nontrivial $k \omega$-nodes.

Importantly according to our studies in [FigTWTbk, 13, 30] the nodal velocities are in fact the points of degeneracy of the dispersion relation. In addition to that, any nodal velocity $\mathrm{u}_{0}$ has to be a real number which is an extreme point of characteristic function $\mathscr{D}(u, \bar{\gamma})$. Consequently, $\mathrm{u}_{0}$ has to be an extreme point function $\Omega(u)$ defined by equation (2.32). Based on this, we can assume 
that

$$
\left.\partial_{u} \Omega(u)\right|_{\mathrm{u}_{0}}=0,\left.\quad \partial_{u}^{2} \Omega(u)\right|_{\mathrm{u}_{0}} \neq 0
$$

that is, $\mathrm{u}_{0}$ is a point of degeneracy of second-order of function $\Omega(u)$.

To assess the analytic properties of solutions for the characteristic equation (2.18) in the vicinity of $\mathrm{u}_{0}$, we introduce the following "small" dimensionless parameters, which are useful for our studies of local extrema of function $\Omega(u)$ defined by equations (2.32):

$$
\begin{gathered}
\delta_{u}=\frac{u-\mathrm{u}_{0}}{\mathrm{u}_{0}}, \quad \delta_{\omega}=\frac{\omega-\omega_{0}}{\omega_{0}}, \quad \delta_{k}=\frac{k-k_{0}}{k_{0}}, \quad \kappa=\Re\left\{\delta_{k}\right\}, \\
\eta=\left[\xi_{0}^{-1} \delta_{\omega}\right]^{\frac{1}{2}}=\left[\frac{\omega-\omega_{0}}{\omega_{2}}\right]^{\frac{1}{2}}, \quad \xi_{0}=\frac{\omega_{2}}{\omega_{0}}, \quad \omega_{2}=\left.\frac{\mathrm{u}_{0}^{2}}{2} \partial_{u}^{2} \Omega(u)\right|_{\mathrm{u}_{0}} \neq 0,
\end{gathered}
$$

where the square roots in equation (2.41) are naturally defined up to a factor \pm 1 . Notice that parameter $\xi_{0}$ can positive or negative depending on the sign of $\omega_{2}$. Observe also that $\delta_{k}$ can be expressed in terms of $\eta$ and $\delta_{u}$ by the following formulas:

$$
\begin{gathered}
\delta_{k}=\frac{k-k_{0}}{k_{0}}=\frac{1+\delta_{\omega}}{1+\delta_{u}}-1=\frac{1+\xi_{0} \eta^{2}}{1+\delta_{u}}-1, \\
k=\frac{\omega}{u}, \quad k_{0}=\frac{\omega_{0}}{\mathrm{u}_{0}} .
\end{gathered}
$$

Then using the analyticity of function $\Omega(u)$, we introduce its power series

$$
\begin{gathered}
\Omega(u)=\omega_{0}+\omega_{2} \delta_{u}^{2}\left[1+\sum_{n \geq 3} \Omega_{n} \delta_{u}^{n-2}\right], \quad \delta_{u}=\frac{u-\mathrm{u}_{0}}{\mathrm{u}_{0}}, \\
\omega_{2}=\left.\frac{\mathrm{u}_{0}^{2}}{2} \partial_{u}^{2} \Omega(u)\right|_{\mathrm{u}_{0}} \neq 0, \quad \Omega_{n}=\frac{\mathrm{u}_{0}^{n}}{\omega_{2}} \frac{\left.\partial_{u}^{n} \Omega(u)\right|_{\mathrm{u}_{0}}}{n !}, \quad n \geq 3,
\end{gathered}
$$

where $\omega_{0}$ and $\omega_{2}$ have the physical dimensions of frequency, whereas variable $\delta_{u}$ and coefficients $\Omega_{n}$ are dimensionless. Notice that coefficients $\omega_{0}, \omega_{2}$ and $\Omega_{n}$ are all real.

Using dimensionless variables (2.42) and series (2.44) we can recast equation (2.32) as follows:

$$
\delta_{\omega}=\xi_{0} \delta_{u}^{2}\left[1+\sum_{n \geq 3} \Omega_{n} \delta_{u}^{n-2}\right]
$$

The analysis of equations (2.40)-(2.45) carried out in [FigTWTbk, 13, 54] provides the following power series approximation that relates $\delta_{\omega}$ and $\kappa=\Re\left\{\delta_{k}\right\}$ :

$$
\delta_{\omega}=\frac{\omega-\omega_{0}}{\omega_{0}}=\left\{\begin{array}{rll}
\xi_{0} \kappa^{2}+\cdots, & \text { if } & \xi_{0}^{-1} \delta_{\omega}>0 \\
\frac{2 \xi_{0}}{2+2 \xi_{0}+\Omega_{3}} \kappa+\cdots, & \text { if } & \xi_{0}^{-1} \delta_{\omega}<0
\end{array}, \quad \kappa=\Re\left\{\delta_{k}\right\}=\Re\left\{\frac{k-k_{0}}{k_{0}}\right\} .\right.
$$

Solutions to characteristic equation (2.18) or the equivalent to it equation (2.33) can be transformed into dispersion relation $\omega=\omega(\Re\{k\})$, which is represented graphically in Fig. 7 for the data as in equation (2.31).

\section{Analysis of the TWT at the EPD}

The analysis of the TWT at the EPD we carry out here is based on the TWT properties reviewed in Section 2. In particular, we take a close look into the spectral properties of the TWT at the 


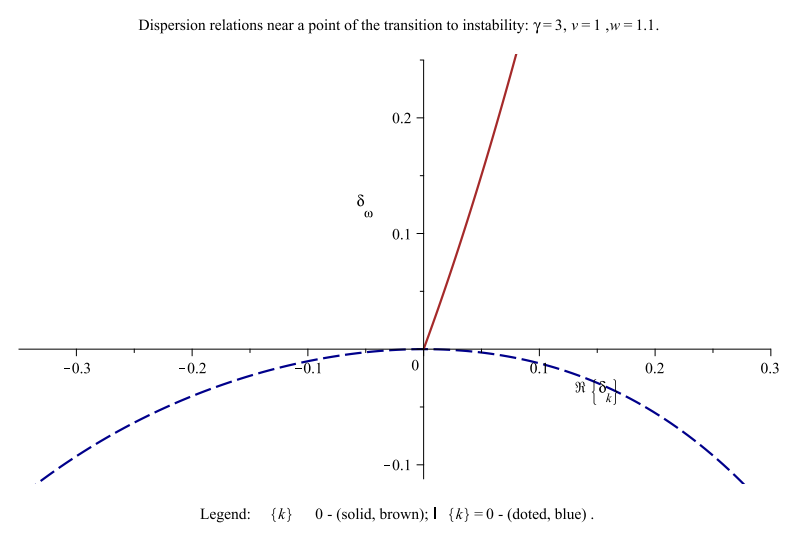

Figure 7. A fragment of the dispersion-instability graph in a vicinity of nodal velocity $\mathbf{u}^{+}(\bar{\gamma})$ computed based on asymptotic formulas (2.46) for $\stackrel{\circ}{*}=1, w=1.1$, $\gamma=3$ (horizontal axis $-\Re\left\{\delta_{k}\right\}$, vertical axis $-\delta_{\omega}$ ). Solid (brown) lines represent unstable branches with $\Im\{k\} \neq 0$, and dotted (blue) lines represent oscillatory branches with $\Im\{k\}=0$.

EPD associated with the nodal velocity $\mathbf{u}^{+}(\bar{\gamma})$ considered in Section 2.3. These properties are related to the TWT monic matrix polynomial $\mathrm{M}_{u \omega}$ defined in equations (2.28), that is

$$
\mathrm{M}_{u \omega}=\left[\begin{array}{cc}
\check{u}^{2}-\chi^{2} & -\chi^{2} \\
\frac{\check{\gamma}}{\check{\omega}^{-2}-1} & \check{u}^{2}+\frac{2 \check{u}}{\check{\omega}^{-2}-1}+\frac{\check{\gamma}-1}{\check{\omega}^{-2}-1}
\end{array}\right] .
$$

for $\check{u}=\mathrm{u}^{+}(\bar{\gamma})$, and to the corresponding companion matrix we consider in Section 3.2. For the reader's convenience the basics of the theory of matrix polynomials and the corresponding companion matrices are reviewed in Section B.

In turns out that it is advantageous for our purposes here to use the nodal velocity

$$
p=\mathrm{u}^{+}(\bar{\gamma})>0
$$

as a new independent variable in place of parameter $\bar{\gamma}$. Using the fact that $\mathbf{u}^{+}(\bar{\gamma})$ is a monotonically decreasing function of $\bar{\gamma}$ we can uniquely recover $\bar{\gamma}$ from $p$ based on equation (3.2). We naturally refer to $p$ and the nodal velocity for: (i) it is a point of degeneracy for the dispersion relations associated with the nodal velocity $\mathbf{u}^{+}(\bar{\gamma})$ and (ii) it is also a point of local minimum of the characteristic function $\mathscr{D}(\check{u}, \check{\gamma})$ defined by equation (2.29), see Figs. 4 (b), 5 and 6 (a) for graphical illustration.

3.1. Nodal velocity as the TWT parameter. Plugging $\check{u}=p$ in the nodal equation (2.36) we obtain the following representation of $\check{\gamma}$ in terms $p$ :

$$
\check{\gamma}=\check{\gamma}_{\mathrm{e}}=\check{\gamma}_{\mathrm{e}}(p, \chi)=\frac{(1-p)\left(p^{2}-\chi^{2}\right)^{2}}{p^{4}}>0, \quad 0<p<1 .
$$

Notice that equation (3.3) can be viewed as the inversion of equation (3.2) that defines the nodal velocity $p=\mathrm{u}^{+}(\bar{\gamma})$. Under assumption that $\chi$ is given and fixed function $\check{\gamma}_{\mathrm{e}}(p, \chi)$ in equation (3.3) relates the nodal velocity $p$ to the TWT principle parameter $\check{\gamma}$. Notice that $\check{\gamma}_{\mathrm{e}}(p, \chi)$ is a monotonically decreasing function of $p$ as illustrated by Figure 8 . The function monotonicity can be established by an examination of the sign of its derivative, which is

$$
\partial_{p}\left(\check{\gamma}_{\mathrm{e}}(p, \chi)\right)=\frac{\left(\chi^{2}-p^{2}\right)\left(3 \chi^{2} p+p^{3}-4 \chi^{2}\right)}{p^{5}}<0, \text { for } 0<p<1<\chi .
$$


Since factor $3 \chi^{2} p+p^{3}-4 \chi^{2}$ in the right-hand side of equation (3.4) is a monotonically growing function of $p$ it satisfies the following inequalities

$$
-4 \chi^{2}<3 \chi^{2} p+p^{3}-4 \chi^{2} \leq 1-\chi^{2}<0, \text { for } 0<p<1<\chi,
$$

implying that $\check{\gamma}_{\mathrm{e}}(p, \chi)$ is indeed a monotonically decreasing function of $p$.

Typical values of the TWT principal parameter $\check{\gamma}$ are small and according to FigTWTbk, Remark 62.1] they vary between $2 \cdot 10^{-6}$ and 0.00675 . Small values of $\check{\gamma}$ according to relation (3.3) correspond to values of the nodal velocity $p$ that are close to 1 , and the following asymptotic formula holds

$$
\check{\gamma}_{\mathrm{e}}(p, \chi)=\left(\chi^{2}-1\right)^{2}(1-p)+O((1-p)), \quad p \rightarrow 1-0 .
$$

Figure 8 shows the graph of function $\check{\gamma}_{\mathrm{e}}(p, \chi)$ as in equation (3.3) for small values of $\check{\gamma}$.

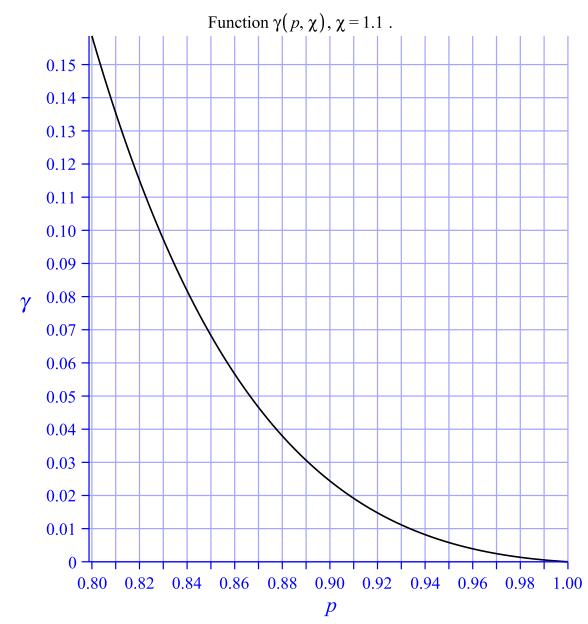

(a)

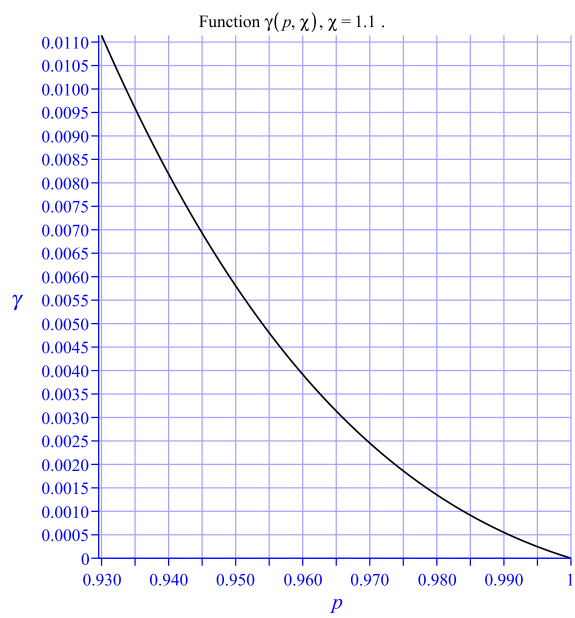

(b)

Figure 8. Plots of function $\check{\gamma}_{\mathrm{e}}(p, \chi)$ as in (3.3) for $\chi=1.1$ and (a) $0.8<p<1$; (b) $0.93<p<1$.

Using equations (2.29) and (3.3) we obtain the following representation of the characteristic function $\mathscr{D}$ and the corresponding characteristic equation:

$$
\mathscr{D}_{\mathrm{e}}(\check{u}, p)=\frac{(p-1)\left(p^{2}-\chi^{2}\right)^{2}}{\left(\check{u}^{2}-\chi^{2}\right) p^{4}}+\frac{(\check{u}-1)^{2}}{\check{u}^{2}}=\frac{1}{\check{\omega}^{2}} .
$$

In particular, plugging in $\check{u}=p$ in $\mathscr{D}(\check{u}, p)$ we obtain

$$
\mathscr{D}_{\mathrm{e}}(p, p)=\frac{(1-p)\left(\chi^{2}-p^{3}\right)}{p^{4}}>0 \text {, for } 0<p<1<\chi .
$$

Hence we can introduce now a positive frequency $\check{\omega}_{\mathrm{e}}=\check{\omega}_{\mathrm{e}}(p, \chi)$ by the following equality

$$
\frac{1}{\check{\omega}_{\mathrm{e}}^{2}}=\mathscr{D}_{\mathrm{e}}(p, p)=\frac{(1-p)\left(\chi^{2}-p^{3}\right)}{p^{4}},
$$

or equivalently

$$
\check{\omega}_{\mathrm{e}}=\check{\omega}_{\mathrm{e}}(p, \chi)=\frac{1}{\sqrt{\mathscr{D}_{\mathrm{e}}(p, p)}}=\frac{p^{2}}{\sqrt{(1-p)\left(\chi^{2}-p^{3}\right)}}>0 \text {, for } 0<p<1<\chi .
$$

We refer to $\check{\omega}_{\mathrm{e}}=\check{\omega}_{\mathrm{e}}(p, \chi)$ as EPD frequency. In view of equations (3.7) and (3.4) the EPD frequency $\check{\omega}_{\mathrm{e}}(p, \chi)$ defined by equations (3.10) corresponds to the nodal velocity $p$. Figure 9 is a 
graphical representation of function $\check{\omega}_{\mathrm{e}}(p, \chi)$ as in equation (3.10) for different ranges of values of the nodal velocity $p$.

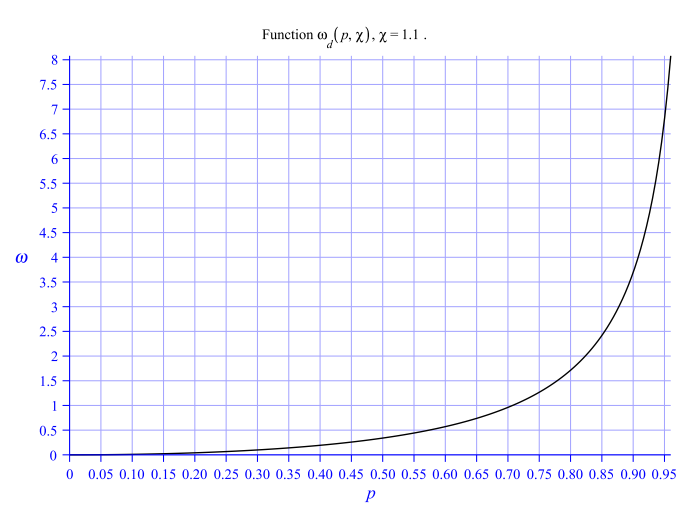

(a)

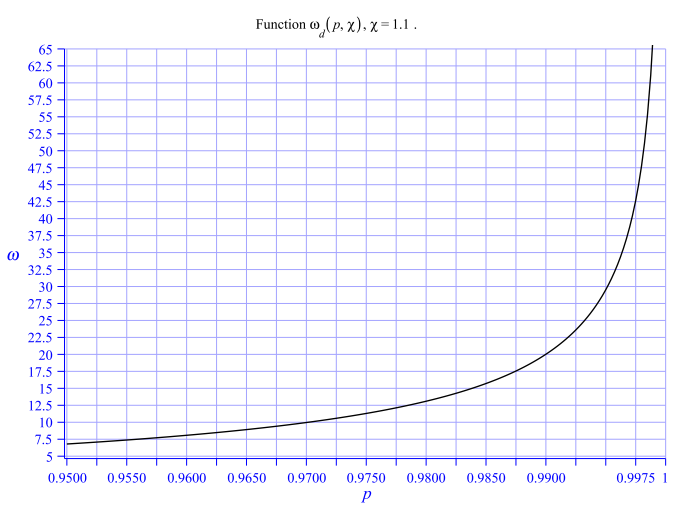

(b)

Figure 9. Plots of function $\check{\omega}_{\mathrm{e}}(p, \chi)$ as in equation (3.10) for $\chi=1.1$ and (a) $0<p<0.95$; (b) $0.95<p<1$.

Using the EPD frequency $\check{\omega}_{\mathrm{e}}(p, \chi)$ defined by equations (3.10) we obtain the following equivalent form of the characteristic equation (3.7)

$$
\begin{gathered}
\mathscr{D}_{\mathrm{e}}(\check{u}, p)=\mathscr{D}_{\mathrm{e}}(\check{u}, p)-\frac{1}{\check{\omega}_{\mathrm{e}}^{2}(p, \chi)}=\frac{1}{\check{\omega}^{2}}-\frac{1}{\check{\omega}_{\mathrm{e}}^{2}(p, \chi)}, \text { where } \\
\mathscr{D}_{\mathrm{e}}(\check{u}, p)=\frac{(\check{u}-p)^{2}\left[\left(\chi^{2} p+p^{3}-\chi^{2}\right) \check{u}^{2}+2 \chi^{2} p(p-1) \check{u}-p^{2} \chi^{2}\right]}{\check{u}^{2} p^{4}\left(\check{u}^{2}-\chi^{2}\right)} .
\end{gathered}
$$

It is evident that for $\check{\omega}=\breve{\omega}_{\mathrm{e}}$ velocity $\check{u}=p$ is a solution to equation (3.11) of multiplicity 2 . We refer to equation (3.11) as the EPD form of the characteristic equation. An algebraic factorized form of rational function $\mathscr{D}_{\mathrm{e}}(\check{u}, p)$ defined by equations (3.11) is

$$
\mathscr{D}_{\mathrm{e}}(\check{u}, p)=\frac{\left(\chi^{2} p+p^{3}-\chi^{2}\right)(\check{u}-p)^{2}\left(\check{u}-\lambda_{+}(p)\right)\left(\check{u}-\lambda_{+}(p)\right)}{\check{u}^{2} p^{4}\left(\check{u}^{2}-\chi^{2}\right)},
$$

where

$$
\lambda_{ \pm}(p)=\frac{p \chi\left[\chi(1-p) \pm \sqrt{p^{3}-p \chi^{2}(1-p)}\right]}{p^{3}-(1-p) \chi^{2}} .
$$

We infer based on the above analysis that for any given $p$ and $\chi$ the EPD values of the original TWT dimensionless parameters are

$$
\operatorname{EPD}: \check{u}=p, \quad \check{\gamma}=\check{\gamma}_{\mathrm{e}}(p, \chi)=\frac{(1-p)\left(p^{2}-\chi^{2}\right)^{2}}{p^{4}}, \quad \check{\omega}=\check{\omega}_{\mathrm{e}}(p, \chi)=\frac{p^{2}}{\sqrt{(1-p)\left(\chi^{2}-p^{3}\right)}} .
$$

3.2. Companion matrix spectral analysis. According to our review on matrix polynomials and associated with them companion matrices in Section B the companion matrix associated with matrix polynomial $\mathrm{M}_{u \omega}$ as in equation (3.1) takes the form

$$
\mathscr{C}=\left[\begin{array}{rrrr}
0 & 0 & 1 & 0 \\
0 & 0 & 0 & 1 \\
\chi^{2} & \chi^{2} & 0 & 0 \\
-\frac{\check{\gamma}}{\check{\omega}^{-2}-1} & -\frac{\check{\gamma}-1}{\check{\omega}^{-2}-1} & 0 & -\frac{2}{\check{\omega}^{-2}-1}
\end{array}\right] .
$$


If $\check{u}$ is a characteristic velocity and consequently an eigenvalue of companion matrix $\mathscr{C}$ then its unique eigenvector $Y(\check{u})$ is defined by the following expression

$$
Y(\check{u})=\left[\begin{array}{r}
\chi^{2} \\
\check{u}^{2}-\chi^{2} \\
\chi^{2} \check{u} \\
\check{u}\left(\check{u}^{2}-\chi^{2}\right)
\end{array}\right]
$$

Notice that expression (3.1) for the eigenvector $Y(\check{u})$ of companion matrix $\mathscr{C}$ depends remarkably on parameter $\chi$ and the value of the corresponding eigenvalue $\check{u}$ of $\mathscr{C}$ only.

The value $\mathscr{C}_{\mathrm{e}}$ of companion matrix $\mathscr{C}$ at the EPD point $\check{u}=p$ can be obtained by plugging in its expression (3.1) the EPD values of $\check{\gamma}=\check{\gamma}_{\mathrm{e}}$ and $\check{\omega}=\check{\omega}_{\mathrm{e}}$ defined in equations (3.14) resulting in

$$
\mathscr{C}_{\mathrm{e}}=\left[\begin{array}{rrrr}
0 & 0 & 1 & 0 \\
0 & 0 & 0 & 1 \\
\chi^{2} & \chi^{2} & 0 & 0 \\
\frac{(1-p)\left(p^{2}-\chi^{2}\right)^{2}}{p^{3}-(1-p) \chi^{2}} & \frac{(1-p) \chi^{2}\left(\chi^{2}-2 p^{2}\right)-p^{5}}{p^{3}-(1-p) \chi^{2}} & 0 & \frac{2 p^{4}}{p^{3}-(1-p) \chi^{2}}
\end{array}\right]
$$

Elementary but tedious analysis shows the Jordan canonical form $\mathscr{J}_{\text {e }}$ of companion matrix $\mathscr{C}_{\mathrm{e}}$ is

$$
\mathscr{J}_{\mathrm{e}}=\left[\begin{array}{rrrr}
p & 1 & 0 & 0 \\
0 & p & 0 & 0 \\
0 & 0 & \lambda_{+}(p) & 0 \\
0 & 0 & 0 & \lambda_{-}(p)
\end{array}\right], \quad \lambda_{ \pm}(p)=\frac{p \chi\left[\chi(1-p) \pm \sqrt{p^{3}-p \chi^{2}(1-p)}\right]}{p^{3}-(1-p) \chi^{2}}
$$

Notice that expressions for eigenvalues $\lambda_{ \pm}(p)$ are consistent with equations (3.12) and (3.13) as one may expect.

The Jordan basis of the generalized eigenspace of the EPD companion matrix $\mathscr{C}_{\mathrm{e}}$ is formed by the eigenvector $Y_{\mathrm{e}}(p)$ and the so-called root vector $Y_{\mathrm{e}}^{\prime}(p)$ which are as follows

$$
Y_{\mathrm{e}}(p)=\left[\begin{array}{r}
\chi^{2} \\
p^{2}-\chi^{2} \\
\chi^{2} p \\
p\left(p-\chi^{2}\right)
\end{array}\right], \quad Y_{\mathrm{e}}^{\prime}(p)=\partial_{p} Y_{\mathrm{e}}(p)=\left[\begin{array}{r}
0 \\
2 \check{u} \\
\chi^{2} \\
3 \check{u}^{2}-\chi^{2}
\end{array}\right]
$$

where root vector $Y_{\mathrm{e}}^{\prime}(p)$ satisfies the following relations

$$
\left(\mathscr{C}_{\mathrm{e}}-p \mathbb{I}\right) Y_{\mathrm{e}}^{\prime}(p)=Y_{\mathrm{e}}(p), \quad\left(\mathscr{C}_{\mathrm{e}}-p \mathbb{I}\right)^{2} Y_{\mathrm{e}}^{\prime}(p)=0
$$

The fact that $Y_{\mathrm{e}}^{\prime}(p)=\partial_{p} Y_{\mathrm{e}}(p)$ is of course not incidental. It can be argued based on the fact that the eigenvector $Y(\check{u})$ of companion matrix $\mathscr{C}$ defined by equation (3.16) depends on parameter $\chi$ and the value of the corresponding eigenvalue $\check{u}$ of $\mathscr{C}$ only. But regardless to the argument one can verify the validity of equations (3.20) by tedious by straightforward evaluation.

Then using (i) the Jordan basis formed by vectors $Y_{\mathrm{e}}(p)$ and $Y_{\mathrm{e}}^{\prime}(p)$ defined by equations (3.19) and (ii) the general expression (3.16) for the eigenvector $Y(\breve{u})$ of companion matrix $\mathscr{C}$ we obtain complete Jordan basis for the EPD companion matrix $\mathscr{C}_{\mathrm{e}}$ formed by columns of the following matrix

Consequently, we have

$$
\mathscr{Y}=\left[Y_{\mathrm{e}}(p), Y_{\mathrm{e}}^{\prime}(p), Y\left(\lambda_{+}(p)\right), Y\left(\lambda_{-}(p)\right)\right]
$$

$$
\mathscr{C}_{\mathrm{e}}=\mathscr{Y} \mathscr{J}_{\mathrm{e}} \mathscr{Y}^{-1}
$$

where matrices $\mathscr{J}_{\mathrm{e}}$ and $\mathscr{Y}$ are defined respectively by equations (3.18) and (3.21).

As to physical significance of the EPD phase velocity $p$ and the EPD frequency $\check{\omega}_{\mathrm{e}}$ they can be detected and identified by their intrinsic association with the onset of instability. Indeed, gradually increasing frequency $\omega$ of probing excitation of the TWT one can detect its value $\check{\omega}_{\mathrm{e}}$ when the 
instability sets up. At this point one can also assess the value of the EPD phase velocity $p$ by comparing time dependent input and output signals. Using then equations (3.14) and assuming that $p$ and $\check{\omega}_{\mathrm{e}}$ are known we recover the TWT model parameters as follows:

$$
\chi^{2}=p^{3}\left(1+\frac{p}{(1-p) \check{\omega}_{\mathrm{e}}}\right), \quad \check{\gamma}=\frac{\left[p^{2}-\check{\omega}_{\mathrm{e}}^{2}(1-p)^{2}\right]^{2}}{(1-p) \check{\omega}_{\mathrm{e}}^{4}} .
$$

\section{USING AN EPD FOR ENHANCED SENSING OF SMALL SIGNALS}

Based on our studies in Section 3 we develop here an approach for using the EPD of the TWT for enhanced sensing of small signals. This approach has some similarity to what we advanced in FigPert for simple circuits with EPDs but it is naturally somewhat more complex. We remind that our primary motivation for considering the TWT is that it can operate at much higher frequencies compare to frequencies for lumped circuits.

Since TWTs are used mostly as amplifiers with the gain varying exponentially with their length one might entertain an idea that the exponential amplification can be exploited for sensing of small signals. The problem with this idea though is that the origin of TWT amplification is an instability and that is hardly compatible with enhanced sensing of small signals. In addition to that, in the case of exponential amplification a variety of noises that naturally occur in any TWT can obscure the small sensor signal.

An EPD in a TWT is in fact also associated with an instability but the EPD regime is at least marginally stable. In particular, if we choose TWT regime to be near the EPD rather than exactly at it the TWT operation can be stable as we showed in [FigPert. This approach is of course a trade off allowing to buy the stability in exchange for reduced value of the enhancement factor for the small sensed signal. But even with such a trade off in place one can get more than 100 fold enhancement FigPert.

4.1. Mathematical model for sensing. We start with equations (2.2) and (2.4) that relate the TWT system model parameters $\chi$ and $\check{\gamma}$ to the primary physical quantities, namely

$$
\chi=\frac{w}{\dot{v}}=\frac{1}{\dot{v} \sqrt{C L}}, \quad \check{\gamma}=\frac{\gamma}{\dot{v}^{2}}=\frac{K_{\gamma}}{C}, \quad K_{\gamma}=\frac{b^{2}}{\dot{v}^{2}} \frac{e^{2}}{m} R_{\mathrm{sc}}^{2} \sigma_{\mathrm{B}} \stackrel{\circ}{n} .
$$

We proceed then with an assumption that the e-beam parameters $\stackrel{\circ}{,} \sigma_{\mathrm{B}}$ and $\stackrel{\circ}{n}$ are chosen, fixed and maintain their values through the process of sensing. We suppose further that: (i) it is either the distributed capacitance $C$ or the distributed inductance $L$ utilized for sensing; (ii) parameter $C$ or $L$ which is selected for sensing is slightly altered by the small sensor signal. The relevant alteration caused by sensed signal is assessed by measuring the relevant characteristic velocities $\breve{u}$ of the TWT. These velocities $\check{u}$ are solutions to the EPD form (3.11) of the characteristic equation, that is

$$
\frac{(\check{u}-p)^{2}\left[\left(\chi^{2} p+p^{3}-\chi^{2}\right) \check{u}^{2}+2 \chi^{2} p(p-1) \check{u}-p^{2} \chi^{2}\right]}{\check{u}^{2} p^{4}\left(\check{u}^{2}-\chi^{2}\right)}=\frac{1}{\check{\omega}^{2}}-\frac{1}{\check{\omega}_{\mathrm{e}}^{2}(p, \chi)},
$$

where parameters $p$ and $\check{\omega}_{\mathrm{e}}$ are related to parameters $\check{\gamma}$ and $\chi$ by equations (3.23). More precisely, with the equation (4.2) in mind we probe the TWT at frequency $\check{\omega}$ of our choosing and then measure the phase velocities $\check{u}$ of the excited eigenmodes of the TWT system. Since according to our TWT model these velocities $\breve{u}$ satisfy characteristic equation (4.2) we can relate them to values of parameters $p$ and $\chi$. Having found $p$ and $\chi$ we can recover then the values of $C$ and $L$ based on equations (3.23) and (4.1) as we show below.

To have a clarity on what kind of the TWT states are considered. We remind that the TWT significant properties are encoded in its companion matrix $\mathscr{C}$ defined by equation (3.15). This matrix in turn is determined by parameters $\check{\gamma}, \chi$ and frequency $\check{\omega}$. Instead of parameter $\check{\gamma}$ we can 
use the nodal velocity $p$ related to it by equation (3.3). Then based on the mentioned quantities we introduce the following definitions of the TWT configuration and TWT state.

Definition 1 (TWT configuration and state). TWT configuration is defined as a pair of two dimensionless TWT parameters: (i) $\chi=\frac{w}{v}$; (ii) the nodal velocity $p=p(\chi, \check{\gamma})$ satisfying equation (3.14). TWT state is defined as a triple of o dimensionless parameters: $\chi, p$ and $\check{\omega}$. Frequency $\check{\omega}$ can be viewed as a parameter that selects the corresponding four TWT eigenmodes associated with the TWT companion matrix $\mathscr{C}$ defined by equation (3.15). The selection is facilitated physically by exciting/probing the TWT at frequency $\check{\omega}$. An EPD state is defined by a particular choice of its parameters, namely $\chi, p$ and $\check{\omega}=\check{\omega}_{\mathrm{e}}(p, \chi)$ where the EPD frequency $\check{\omega}_{\mathrm{e}}(p, \chi)$ is defined by equations (3.10). Work point state is defined by the following choice of its parameters $\chi, p$ and $\check{\omega}=\check{\omega}_{\mathrm{w}}<\check{\omega}_{\mathrm{e}}(p, \chi)$. We refer to $\check{\omega}_{\mathrm{w}}$ as work point frequency. There is a flexibility in choosing $\check{\omega}_{\mathrm{w}}$ to be proximate to the EPD frequency $\breve{\omega}_{\mathrm{e}}(p, \chi)$ when at the same time to maintain certain distance from it to provide for the stability of the TWT operation as explained in Remark 2. There are exactly two eigenmodes with their phase velocities $\breve{u}$ close to $p$ that are of particular significance.

Remark 2 (work point). It turns out that the ideal EPD state is intrinsically only marginally stable and the purpose of the work point is to overcome this problem. The stability of the work point is achieved by making a deliberate small departure from the EPD frequency $\check{\omega}_{\mathrm{e}}$. The departure is achieved by appropriate selection of the probing frequency $\check{\omega}=\check{\omega}_{\mathrm{w}}$ so that $\check{\omega}_{\mathrm{w}}<\check{\omega}_{\mathrm{e}}$. An additional benefit and utility of the work point frequency $\check{\omega}_{\mathrm{w}}$ is that it lifts the characteristic velocity degeneracy causing the velocity split. This velocity split can be measured and used to determine the small sensed signal, see Theorem [3, Fig. 10 and equations (4.23) and (4.24).

Suppose that the TWT configuration before sensing is defined by parameters $\chi$ and $p$ and the TWT configuration altered by the small sensed signal is defined by parameters $\chi^{\prime}$ and $p^{\prime}$. We proceed then with introducing a larger set of parameters associated with the EPD state of the TWT before it it receives the small sensed signal:

$$
\text { EPD state: } C, \quad L, \quad p, \quad \chi, \quad \check{\omega}=\check{\omega}_{\mathrm{e}}(p, \chi) \text {. }
$$

Using the above EPD state as a reference point we introduce a larger set of parameters for altered $E P D$ state associated with the small sensed signal:

$$
\begin{aligned}
& \text { altered EPD state: } C^{\prime}=C\left(1+\delta_{C}\right), \quad L^{\prime}=L\left(1+\delta_{L}\right), \quad p^{\prime}=p\left(1+\delta_{p}\right), \\
& \left(\chi^{2}\right)^{\prime}=\chi^{2}\left(\check{1}+\delta_{\chi^{2}}\right), \quad \omega=\check{\omega}_{\mathrm{e}}^{\prime}=\check{\omega}_{\mathrm{e}}\left(p^{\prime}, \chi^{\prime}\right)=\check{\omega}_{\mathrm{e}}\left(1+\delta_{\omega}\right), \quad \delta_{\omega}=\frac{\check{\omega}_{\mathrm{e}}^{\prime}-\check{\omega}_{\mathrm{e}}}{\check{\omega}_{\mathrm{e}}},
\end{aligned}
$$

where as a matter of computational convenience we use parameter $\chi^{2}$ rather than $\chi$. In relations (4.3)-(4.7) the relative variation coefficients $\delta_{*}$ are assumed to be small and satisfy the following relations

$$
\left|\delta_{C}\right|,\left|\delta_{L}\right|,\left|\delta_{\chi^{2}}\right|,\left|\delta_{p}\right| \ll 1 ; \quad\left|\delta_{\omega}\right| \ll\left|\delta_{\mathrm{w}}\right| \ll 1 .
$$

Using once again the EPD state as a reference point we introduce parameters of the work point state and its altered version as follows:

$$
\text { work point state: } C, \quad L, \quad p, \quad \chi, \quad \check{\omega}=\check{\omega}_{\mathrm{w}}=\check{\omega}_{\mathrm{e}}\left(1+\delta_{\mathrm{w}}\right), \quad \delta_{\mathrm{w}}=\frac{\check{\omega}_{\mathrm{w}}-\check{\omega}_{\mathrm{e}}}{\check{\omega}_{\mathrm{e}}} \text {, }
$$

altered work point state: $C^{\prime}=C\left(1+\delta_{C}\right), \quad L^{\prime}=L\left(1+\delta_{L}\right), \quad p^{\prime}=p\left(1+\delta_{p}\right)$,

$$
\left(\chi^{2}\right)^{\prime}=\chi^{2}\left(1+\delta_{\chi^{2}}\right), \check{\omega}=\check{\omega}_{\mathrm{w}}=\check{\omega}_{\mathrm{e}}\left(1+\delta_{\mathrm{w}}\right), \check{\omega}_{\mathrm{e}}^{\prime}=\check{\omega}_{\mathrm{e}}\left(p^{\prime}, \chi^{\prime}\right)=\check{\omega}_{\mathrm{e}}\left(1+\delta_{\omega}\right), \delta_{\omega}=\frac{\check{\omega}_{\mathrm{e}}^{\prime}-\check{\omega}_{\mathrm{e}}}{\check{\omega}_{\mathrm{e}}} \text {. }
$$


Relative incremental frequency differences $\delta_{\mathrm{w}}$ and $\delta_{\omega}$ employ effectively the EPD frequency $\check{\omega}_{\mathrm{e}}$ as a natural frequency unit.

Notice that parameters in relations (4.3)-(4.7) are not independent but rather parameters $\chi$, $p$ and consequently $\check{\omega}_{\mathrm{e}}$ can be expressed in terms of the primary physical parameters $C$ and $L$. Namely, according to relations (3.14) and (4.1) we have

$$
\chi=\frac{1}{\grave{v} \sqrt{C L}}, \quad \check{\gamma}=\frac{K_{\gamma}}{C}=\frac{(1-p)\left(p^{2}-\chi^{2}\right)^{2}}{p^{4}}, \quad \check{\omega}_{\mathrm{e}}=\frac{p^{2}}{\sqrt{(1-p)\left(\chi^{2}-p^{3}\right)}} .
$$

Since in view of made assumptions the e-beam parameters $\stackrel{i}{v}$ and $K_{\gamma}$ can be considered to be constants the first two equations in (4.8) allow to express $\chi$ and $p$ as a functions of $C$ and $L$. Consequently the third equation in (4.8) determines $\breve{\omega}_{\mathrm{e}}$ as a function of $C$ and $L$ also.

We proceed now with the derivation of linear approximations, that is the differentials, for $\delta_{\chi^{2}}$, $\delta_{p}$ and $\delta_{\omega}$ in terms of $\delta_{C}$ and $\delta_{L}$. The assumed smallness of $\delta_{C}, \delta_{L}$ and $\delta_{\omega}$ imply the smallness of $\delta_{\chi^{2}}, \delta_{p}$ and $\delta_{\omega}^{\prime}$. Hence the work point and the altered work point states are all close to the EPD state. Using the proximity of all these states we obtain the following first order approximation to the characteristic equation (4.2) for the altered state

$$
\left(\check{u}-p^{\prime}\right)^{2} \cong S\left(\check{\omega}_{\mathrm{e}}^{\prime}-\check{\omega}_{\mathrm{w}}\right)=S \check{\omega}_{\mathrm{e}}\left(\delta_{\omega}-\delta_{\mathrm{w}}\right),
$$

where

$$
\begin{gathered}
\delta_{\omega}=\frac{\check{\omega}_{\mathrm{e}}^{\prime}-\check{\omega}_{\mathrm{e}}}{\check{\omega}_{\mathrm{e}}}, \quad \delta_{\mathrm{w}}=\frac{\check{\omega}_{\mathrm{w}}-\check{\omega}_{\mathrm{e}}}{\check{\omega}_{\mathrm{e}}}, \quad \check{\omega}_{\mathrm{e}}=\check{\omega}_{\mathrm{e}}(p, \chi), \quad \check{\omega}_{\mathrm{e}}^{\prime}=\check{\omega}_{\mathrm{e}}\left(p^{\prime}, \chi^{\prime}\right), \\
S=S(p, \chi)=\frac{2 p^{4}\left(\chi^{2}-p^{2}\right)\left[(1-p)\left(\chi^{2}-p^{3}\right)\right]^{\frac{3}{2}}}{p^{2}\left(4 \chi^{2}-3 \chi^{2} p-p^{3}\right)}>0, \quad 0<p<1, \quad \chi>1 .
\end{gathered}
$$

We refer to equation (4.9) as EPD approximation to the characteristic equation, and we refer to quantities $\delta_{\omega}$ and $\delta_{\mathrm{w}} \Delta^{\prime}$ and $\Delta_{w}$ respectively as relative EPD increment and relative work point increment. The EPD approximation to the characteristic equation which is a quadratic equation selects two characteristic velocities out of the total of four by their property to be proximate to the $E P D$ velocity $p$. Equations (4.9) readily imply the following statement.

Theorem 3 (velocity split near the EPD). . The velocities $\check{u}_{ \pm}$that solve the quadratic in $\check{u}$ equation (4.9) are

$$
\check{u}_{ \pm}=p^{\prime} \pm \sqrt{S\left(\check{\omega}_{\mathrm{e}}^{\prime}-\check{\omega}_{\mathrm{w}}\right)}=p^{\prime} \pm \sqrt{S \check{\omega}_{\mathrm{e}}\left(\delta_{\omega}-\delta_{\mathrm{w}}\right)},
$$

where factor $S>0$ satisfies relations (4.11) and frequency $\check{\omega}_{\mathrm{e}}>0$ satisfies the last equation in (4.8). The parameters $\chi$ and $p$ that determine $S$ and $\check{\omega}_{\mathrm{e}}$ are associated with the EPD state as in (4.3). Since $S>0$ velocities $\check{u}_{ \pm}$defined by equation (4.12) are real if and only if

$$
\check{\omega}_{\mathrm{w}}<\check{\omega}_{\mathrm{e}}^{\prime}
$$

Equations (4.12) imply the following representation for the velocity split $\check{u}_{+}-\check{u}_{-}$at the EPD:

$$
\begin{aligned}
\check{u}_{+}-\check{u}_{-} & =2 \sqrt{S\left(\check{\omega}_{\mathrm{e}}^{\prime}-\check{\omega}_{\mathrm{w}}\right)}=2 \sqrt{S \check{\omega}_{\mathrm{e}}\left(\delta_{\omega}-\delta_{\mathrm{w}}\right)}, \\
\delta_{\omega} & =\frac{\check{\omega}_{\mathrm{e}}^{\prime}-\check{\omega}_{\mathrm{e}}}{\check{\omega}_{\mathrm{e}}}, \quad \delta_{\mathrm{w}}=\frac{\check{\omega}_{\mathrm{w}}-\check{\omega}_{\mathrm{e}}}{\check{\omega}_{\mathrm{e}}},
\end{aligned}
$$

where frequencies $\check{\omega}_{\mathrm{e}}$, $\check{\omega}_{\mathrm{e}}^{\prime}$ and $\check{\omega}_{\mathrm{w}}$ are associated respectively with the EPD state, the altered state and the work point state defined by equations (4.3), (4.6) and (4.7).

If the following inequalities hold

$$
\left|\delta_{\omega}\right|<\left|\delta_{\mathrm{w}}\right| \text { and }-\delta_{\mathrm{w}}>0,
$$




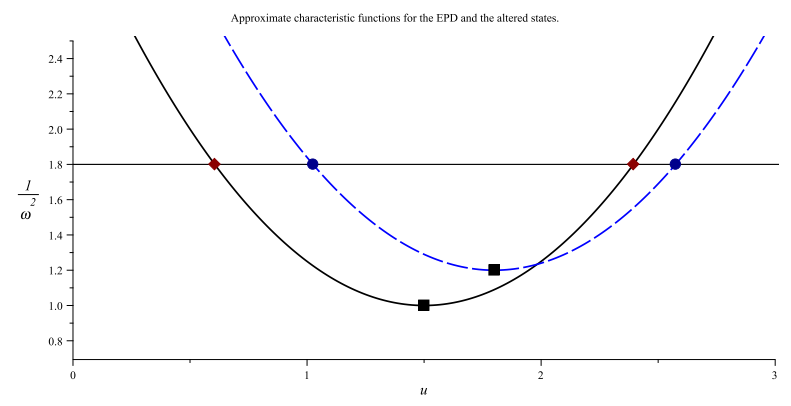

FiguRE 10. An illustrative plot for the approximate characteristic equation similar (4.9) for EPD state represented by black solid parabola and the altered state represented by blue dashed parabola (horizontal axis $-u$, vertical axis $-\frac{1}{\breve{\omega}^{2}}$ ). The black horizontal line corresponds to the work frequency $\check{\omega}=\check{\omega}_{\mathrm{w}}$. The points of intersection of the horizontal line and blue dashed parabola shown as blue disk points represent solutions $\check{u}_{ \pm}$to equation (4.12). The points of intersection of the horizontal line and black solid parabola are shown as brown diamond points represents solutions $\check{u}_{ \pm}$to equation similar (4.12) corresponding to $p$ rather than $p^{\prime}$. The EPDs correspond the to vertexes of the parabolas shown as black square points. Notice that the EPD frequencies $\check{\omega}_{\mathrm{e}}$ and $\check{\omega}_{\mathrm{e}}^{\prime}$ associated with the parabolas vertices satisfy the frequency stability conditions (4.17).

then

$$
\delta_{\omega}-\delta_{\mathrm{w}}=\frac{\check{\omega}_{\mathrm{e}}^{\prime}-\check{\omega}_{\mathrm{w}}}{\check{\omega}_{\mathrm{e}}}>0
$$

readily implying the inequality 4.13).

Remark 4 (stability and square root enhancement factor). We refer to inequalities

$$
\check{\omega}_{\mathrm{w}}<\check{\omega}_{\mathrm{e}}, \check{\omega}_{\mathrm{e}}^{\prime}
$$

that appear in the statement of Theorem 3 as the frequency stability conditions. These conditions are relevant to the stability for they imply the real-valuedness of the velocities $\check{u}_{ \pm}$. Note that inequalities (4.15) imply frequency stability conditions (4.17).

Note also that according to equation (4.14) the velocity split $\check{u}_{+}-\check{u}_{-}$, a quantity that can be measured, is proportional to $\sqrt{\breve{\omega}_{\mathrm{e}}^{\prime}-\check{\omega}_{\mathrm{w}}}$. The square root operation applied to the small frequency difference $\check{\omega}_{\mathrm{e}}^{\prime}-\check{\omega}_{\mathrm{w}}$ effectively "magnifies" it. This is a typical manifestation of the proximity to the EPD. The rise of the square root operation can be traced to the EPD approximation to the characteristic equation (4.9) which is a quadratic in $\check{u}$ equation.

The EPD approximation to the characteristic equation (4.9) and its solutions $\check{u}_{ \pm}$are illustrated by Fig. 10.

4.2. Sensing algorithm. Our approach to utilize the TWT for sensing is as follows. First of all, we assume that (i) the small sensed signal alters either distributed capacitance $C$ or distributed inductance $L$; (ii) the physical alteration of the TWT indicated in (i) is described mathematically by relations (4.3)-(4.5). Second of all, we assume that prior to any measurements the values of parameters $C, L, \chi$ and $p$ that determine the current state of the TWT are established and known. These values constitute a reference point for an assessment of the results of sensing. Third of all, we assume that the relationship between $\delta_{C}$ or $\delta_{L}$ and the small sensed signal is known and is an integral part of the sensor.

The consecutive steps of our sensing algorithm are as follows: 
(i) Apply the small sensed signal to the relevant component of the TWT. Probe then the TWT by an excitation at the work point frequency $\check{\omega}=\check{\omega}_{\mathrm{w}}$.

(ii) Observe the two TWT eigenmodes and assess the corresponding to them characteristic velocities $\breve{u}_{+}$and $\breve{u}_{-}$that are close to the EPD velocity $p$ and satisfy the EPD approximation (4.9) to the characteristic equation.

(iii) Compute the velocity split $\check{u}_{+}-\check{u}_{-}$. Depending on what of the parameters $C$ or $L$ was utilized for sensing find the corresponding value $\delta_{C}$ or $\delta_{L}$ using respectively formulas (4.23) and (4.24) below.

(iv) Based on assumed to be known relationship between $\delta_{C}$ or $\delta_{L}$ and the small sensed signal recover the value of the small sensed signal.

Fig. 10 illustrates graphically the proposed sensing approach. The sensing approach utilizing the EPD of the TWT is conceptually similar to the one for circuits advanced in FigPert but it is naturally more complex. The reason for complexity is that the TWT as a physical system is naturally a more complex system compare to the simple circuits with EPDs we advanced in FigPert.

In the light of Theorem 3 and Remark 4 let us take a closer look at the relative EPD increment $\delta_{\omega}$ and the relative work point increment $\delta_{\mathrm{w}}$ defined by equations (4.10). Both relative increments $\delta_{\omega}$ and $\delta_{\mathrm{w}}$ in equations (4.10) use the EPD frequency $\check{\omega}_{\mathrm{e}}=\check{\omega}_{\mathrm{e}}(p, \chi)$ as a reference point and effectively as a natural frequency unit. The stability of the TWT operation requires the characteristic velocities $\check{u}_{ \pm}$defined by equations (4.12) to be real-valued. This requirement is fulfilled always if and only if the expression under square root in the right-hand side of equation (4.9) is non-negative. That in turn leads to

$$
\delta_{\omega}-\delta_{\mathrm{w}}=\frac{\check{\omega}_{\mathrm{e}}^{\prime}-\check{\omega}_{\mathrm{w}}}{\check{\omega}_{\mathrm{e}}}>0 \text {, implying } \check{\omega}_{\mathrm{w}}<\check{\omega}_{\mathrm{e}}
$$

since $\delta_{\omega}$ can be zero and factors $\check{\omega}_{\mathrm{e}}, S>0$ according to relations (4.8) and (4.11). The second inequality in (4.18) is one of the frequency stability conditions (4.17). As it is indicated previously in the last relations in (4.5) we require the following relations to hold

$$
\left|\delta_{\omega}\right|=\left|\frac{\check{\omega}_{\mathrm{e}}^{\prime}-\check{\omega}_{\mathrm{e}}}{\check{\omega}_{\mathrm{e}}}\right| \ll\left|\delta_{\mathrm{w}}\right|=\left|\frac{\check{\omega}_{\mathrm{w}}-\check{\omega}_{\mathrm{e}}}{\check{\omega}_{\mathrm{e}}}\right| \ll 1 .
$$

The point of inequalities (4.19) as a requirement is to assure that the altered EPD frequency $\check{\omega}_{\mathrm{e}}^{\prime}$ satisfies the frequency stability conditions (4.17) as soon as the EPD frequency $\breve{\omega}_{\mathrm{e}}$ satisfies them. Consequently relations (4.19) assure the effectiveness and robustness of described above approach to sensing.

We proceed now with relating the relative increments $\delta_{\omega}$ and $\delta_{\mathrm{w}}$ defined by equations (4.10) to quantities $\delta_{C}$ and $\delta_{L}$ that are directly effected by the small sensor signal. Using equations (4.8) and assuming that $\delta_{C}$ and $\delta_{L}$ are small after tedious but elementary evaluations we find the following first order approximations

$$
\delta_{\chi^{2}} \cong-\left(\delta_{C}+\delta_{L}\right), \quad \delta_{p} \cong-\frac{(1-p)\left[\left(\chi^{2}+p^{2}\right) \delta_{C}+2 \chi^{2} \delta_{L}\right]}{(4-3 p) \chi^{2}-p^{3}} .
$$

Using representation (4.8) for $\check{\omega}_{\mathrm{e}}(p, \chi)$ and equations (4.20) (4.20) under assumption that $\delta_{C}$ and $\delta_{L}$ are small we obtain the following first order approximation

$$
\delta_{\omega}=\frac{\check{\omega}_{\mathrm{e}}\left(p^{\prime}, \chi^{\prime}\right)-\check{\omega}_{\mathrm{e}}(p, \chi)}{\check{\omega}_{\mathrm{e}}(p, \chi)} \cong-\frac{p^{2} \delta_{C}+\chi^{2} \delta_{L}}{2\left(\chi^{2}-p^{3}\right)} .
$$

Fig. 11 is a graphical representations of function the increment $\check{\omega}_{\mathrm{e}}\left(p^{\prime}, \chi^{\prime}\right)-\check{\omega}_{\mathrm{e}}(p, \chi)$ and its first order approximation as in equation (4.21) for ranges of values of relative variation coefficient $\delta_{C}$ when $\delta_{L}=0$. Fig. 12 similarly is a graphical representation of function the increment $\check{\omega}_{\mathrm{e}}\left(p^{\prime}, \chi^{\prime}\right)-$ 
$\breve{\omega}_{\mathrm{e}}(p, \chi)$ and its first order approximation as in equation (4.21) for two ranges of values of the relative variation coefficient $\delta_{L}$ when $\delta_{C}=0$. Figures 11 and 12 indicate that for the chosen values of parameters when the value of relative variation coefficient $\delta_{C}$ or $\delta_{L}$ is under $5 \%$ the simple formula (4.21) yields pretty accurate values compare to the exact value of the increment. If values relative variation coefficient $\delta_{C}$ or $\delta_{L}$ values are below $1 \%$ the approximation values are nearly the same as the exact values.

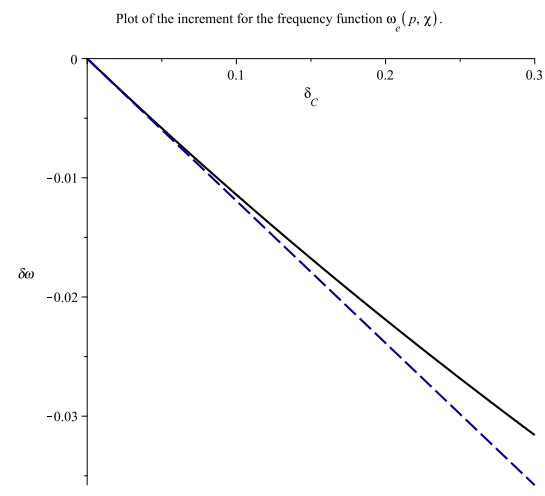

(a)

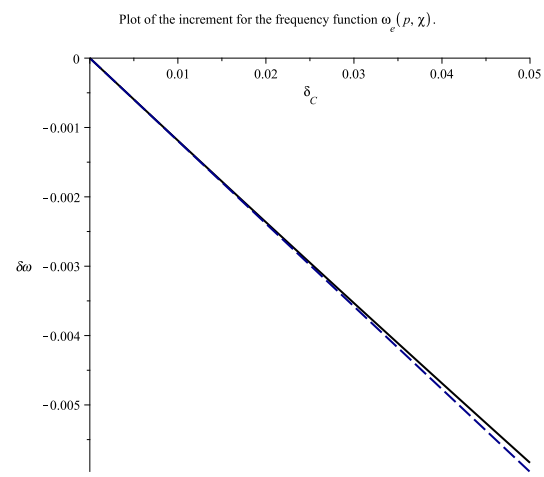

(b)

Figure 11. Plots of the increment $\check{\omega}_{\mathrm{e}}\left(p^{\prime}, \chi^{\prime}\right)-\check{\omega}_{\mathrm{e}}(p, \chi)$ and its first order approximation as in equation (4.21) for $\stackrel{0}{v}=1.1, K_{\gamma}=1.2, C=1.3, L=1.4, \delta_{L}=0$ and: (a) $0<\delta_{C}<0.3$; (b) $0<\delta_{C}<0.05$.

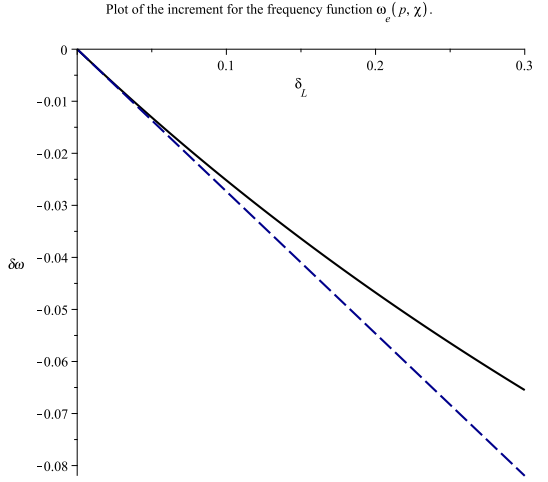

(a)

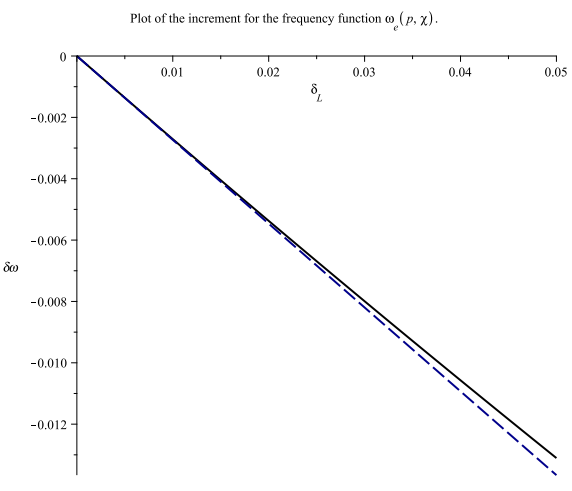

(b)

Figure 12. Plots of the increment $\check{\omega}_{\mathrm{e}}\left(p^{\prime}, \chi^{\prime}\right)-\check{\omega}_{\mathrm{e}}(p, \chi)$ and its first order approximation as in equation (4.21) for $\stackrel{0}{v}=1.1, K_{\gamma}=1.2, C=1.3, L=1.4, \delta_{C}=0$ and: (a) $0<\delta_{L}<0.3$; (b) $0<\delta_{L}<0.05$.

Using equations (4.14) and (4.21) we recover the value of $\delta_{\breve{\omega}}$ based on the measured velocity shift $\check{u}_{+}-\check{u}_{-}$as follows

$$
\delta_{\check{\omega}} \cong-\frac{p^{2} \delta_{C}+\chi^{2} \delta_{L}}{2\left(\chi^{2}-p^{3}\right)}=\frac{\left(\check{u}_{+}-\check{u}_{-}\right)^{2}}{4 S \check{\omega}_{\mathrm{e}}}+\delta_{\mathrm{w}}=\frac{\left(\check{u}_{+}-\check{u}_{-}\right)^{2}}{4 S \check{\omega}_{\mathrm{e}}}+\frac{\check{\omega}_{\mathrm{w}}}{\check{\omega}_{\mathrm{e}}}-1 .
$$

Consequently, if the distributed capacitance $C$ was used for sensing and hence $\delta_{L}=0$ we obtain from equations (4.22) the value of $\delta_{C}$, that is

$$
\delta_{C} \cong \frac{2\left(\chi^{2}-p^{3}\right)}{p^{2}}\left[1-\frac{\check{\omega}_{\mathrm{w}}}{\check{\omega}_{\mathrm{e}}}-\frac{\left(\check{u}_{+}-\check{u}_{-}\right)^{2}}{4 S \check{\omega}_{\mathrm{e}}}\right]
$$


Similarly, if the distributed inductance $L$ was used for sensing and hence $\delta_{C}=0$ we obtain from equations (4.22) the value of $\delta_{L}$, that is

$$
\delta_{L} \cong \frac{2\left(\chi^{2}-p^{3}\right)}{\chi^{2}}\left[1-\frac{\check{\omega}_{\mathrm{w}}}{\check{\omega}_{\mathrm{e}}}-\frac{\left(\check{u}_{+}-\check{u}_{-}\right)^{2}}{4 S \check{\omega}_{\mathrm{e}}}\right] .
$$

\section{Appendix A. Fourier transform}

Our preferred form of the Fourier transforms as in [Foll, 7.2, 7.5], ArfWeb, 20.2]:

$$
\begin{gathered}
f(t)=\int_{-\infty}^{\infty} \hat{f}(\omega) \mathrm{e}^{-\mathrm{i} \omega t} \mathrm{~d} \omega, \quad \hat{f}(\omega)=\frac{1}{2 \pi} \int_{-\infty}^{\infty} f(t) e^{\mathrm{i} \omega t} \mathrm{~d} t \\
f(z, t)=\int_{-\infty}^{\infty} \hat{f}(k, \omega) \mathrm{e}^{-\mathrm{i}(\omega t-k z)} \mathrm{d} k \mathrm{~d} \omega \\
\hat{f}(k, \omega)=\frac{1}{(2 \pi)^{2}} \int_{-\infty}^{\infty} f(z, t) e^{\mathrm{i}(\omega t-k z)} d z \mathrm{~d} t .
\end{gathered}
$$

This preference was motivated by the fact that the so-defined Fourier transform of the convolution of two functions has its simplest form. Namely, the convolution $f * g$ of two functions $f$ and $g$ is defined by [Foll, 7.2, 7.5],

$$
\begin{gathered}
{[f * g](t)=[g * f](t)=\int_{-\infty}^{\infty} f\left(t-t^{\prime}\right) g\left(t^{\prime}\right) \mathrm{d} t^{\prime}} \\
{[f * g](z, t)=[g * f](z, t)=\int_{-\infty}^{\infty} f\left(z-z^{\prime}, t-t^{\prime}\right) g\left(z^{\prime}, t^{\prime}\right) \mathrm{d} z^{\prime} \mathrm{d} t^{\prime} .}
\end{gathered}
$$

Then its Fourier transform as defined by equations (A.1) and (A.2) satisfies the following properties:

$$
\begin{gathered}
\widehat{f * g}(\omega)=\hat{f}(\omega) \hat{g}(\omega), \\
\widehat{f * g}(k, \omega)=\hat{f}(k, \omega) \hat{g}(k, \omega) .
\end{gathered}
$$

\section{Appendix B. MATRIX POLYNOMiAL AND its COMPANiON MATRIX}

An important incentive for considering matrix polynomials is that they are relevant to the spectral theory of the differential equations of the order higher than 1, particularly the EulerLagrange equations which are the second-order differential equations in time. We provide here selected elements of the theory of matrix polynomials following mostly to [GoLaRo, II.7, II.8], [Baum, 9]. General matrix polynomial eigenvalue problem reads

$$
A(s) x=0, \quad A(s)=\sum_{j=0}^{\nu} A_{j} s^{j}, \quad x \neq 0,
$$

where $s$ is complex number, $A_{k}$ are constant $m \times m$ matrices and $x \in \mathbb{C}^{m}$ is $m$-dimensional columnvector. We refer to problem (B.1) of funding complex-valued $s$ and non-zero vector $x \in \mathbb{C}^{m}$ as polynomial eigenvalue problem.

If a pair of a complex $s$ and non-zero vector $x$ solves problem (B.1) we refer to $s$ as an eigenvalue or as a characteristic value and to $x$ as the corresponding to $s$ eigenvector. Evidently the characteristic values of problem (B.1) can be found from polynomial characteristic equation

$$
\operatorname{det}\{A(s)\}=0 \text {. }
$$

We refer to matrix polynomial $A(s)$ as regular if $\operatorname{det}\{A(s)\}$ is not identically zero. We denote by $m\left(s_{0}\right)$ the multiplicity (called also algebraic multiplicity) of eigenvalue $s_{0}$ as a root of polynomial $\operatorname{det}\{A(s)\}$. In contrast, the geometric multiplicity of eigenvalue $s_{0}$ is defined as $\operatorname{dim}\left\{\operatorname{ker}\left\{A\left(s_{0}\right)\right\}\right\}$, 
where $\operatorname{ker}\{A\}$ defined for any square matrix $A$ stands for the subspace of solutions $x$ to equation $A x=0$. Evidently, the geometric multiplicity of eigenvalue does not exceed its algebraic one, see Corollary 7.

It turns out that the matrix polynomial eigenvalue problem (B.1) can be always recast as the standard "linear" eigenvalue problem, namely

$$
(s \mathrm{~B}-\mathrm{A}) \mathrm{x}=0,
$$

where $m \nu \times m \nu$ matrices $\mathrm{A}$ and $\mathrm{B}$ are defined by

$$
\mathrm{B}=\left[\begin{array}{ccccc}
\mathbb{I} & 0 & \cdots & 0 & 0 \\
0 & \mathbb{I} & 0 & \cdots & 0 \\
0 & 0 & \ddots & \cdots & \vdots \\
\vdots & \vdots & \ddots & \mathbb{I} & 0 \\
0 & 0 & \cdots & 0 & A_{\nu}
\end{array}\right], \quad \mathrm{A}=\left[\begin{array}{ccccc}
0 & \mathbb{I} & \cdots & 0 & 0 \\
0 & 0 & \mathbb{I} & \cdots & 0 \\
0 & 0 & 0 & \cdots & \vdots \\
\vdots & \vdots & \ddots & 0 & \mathbb{I} \\
-A_{0} & -A_{1} & \cdots & -A_{\nu-2} & -A_{\nu-1}
\end{array}\right],
$$

with $\mathbb{I}$ being $m \times m$ identity matrix. Matrix A, particularly in monic case, is often referred to as companion matrix. In the case of monic polynomial $A(\lambda)$, when $A_{\nu}=\mathbb{I}$ is $m \times m$ identity matrix, matrix $\mathrm{B}=\mathrm{I}$ is $m \nu \times m \nu$ identity matrix. The reduction of original polynomial problem (B.1) to an equivalent linear problem (B.3) is called linearization.

The linearization is not unique, and one way to accomplish is by introducing the so-called known "companion polynomial" which is $m \nu \times m \nu$ matrix

$$
\mathrm{C}_{A}(s)=s \mathrm{~B}-\mathrm{A}=\left[\begin{array}{ccccc}
s \mathbb{I} & -\mathbb{I} & \cdots & 0 & 0 \\
0 & s \mathbb{I} & -\mathbb{I} & \cdots & 0 \\
0 & 0 & \ddots & \cdots & \vdots \\
\vdots & \vdots & \vdots & s \mathbb{I} & -\mathbb{I} \\
A_{0} & A_{1} & \cdots & A_{\nu-2} & s A_{\nu}+A_{\nu-1}
\end{array}\right]
$$

Notice that in the case of the EL equations the linearization can be accomplished by the relevant Hamilton equations.

To demonstrate the equivalency between the eigenvalue problems for $m \nu \times m \nu$ companion polynomial $\mathrm{C}_{A}(s)$ and the original $m \times m$ matrix polynomial $A(s)$ we introduce two $m \nu \times m \nu$ matrix polynomials $\mathrm{E}(s)$ and $\mathrm{F}(s)$. Namely,

$$
\mathrm{E}(s)=\left[\begin{array}{ccccc}
E_{1}(s) & E_{2}(s) & \cdots & E_{\nu-1}(s) & \mathbb{I} \\
-\mathbb{I} & 0 & 0 & \cdots & 0 \\
0 & -\mathbb{I} & \ddots & \cdots & \vdots \\
\vdots & \vdots & \ddots & 0 & 0 \\
0 & 0 & \cdots & -\mathbb{I} & 0
\end{array}\right]
$$

where $m \times m$ matrix polynomials $E_{j}(s)$ are defined by the following recursive formulas

$$
E_{\nu}(s)=A_{\nu}, \quad E_{j-1}(s)=A_{j-1}+s E_{j}(s), \quad j=\nu, \ldots, 2 .
$$


Matrix polynomial $\mathrm{F}(s)$ is defined by

$$
\mathrm{F}(s)=\left[\begin{array}{ccccc}
\mathbb{I} & 0 & \cdots & 0 & 0 \\
-s \mathbb{I} & \mathbb{I} & 0 & \cdots & 0 \\
0 & -s \mathbb{I} & \ddots & \cdots & \vdots \\
\vdots & \vdots & \ddots & \mathbb{I} & 0 \\
0 & 0 & \cdots & -s \mathbb{I} & \mathbb{I}
\end{array}\right], \quad \operatorname{det}\{\mathrm{F}(s)\}=1
$$

Notice, that both matrix polynomials $\mathrm{E}(s)$ and $\mathrm{F}(s)$ have constant determinants readily implying that their inverses $\mathrm{E}^{-1}(s)$ and $\mathrm{F}^{-1}(s)$ are also matrix polynomials. Then it is straightforward to verify that

$$
\mathrm{E}(s) \mathrm{C}_{A}(s) \mathrm{F}^{-1}(s)=\mathrm{E}(s)(s \mathrm{~B}-\mathrm{A}) \mathrm{F}^{-1}(s)=\left[\begin{array}{ccccc}
A(s) & 0 & \cdots & 0 & 0 \\
0 & \mathbb{I} & 0 & \cdots & 0 \\
0 & 0 & \ddots & \cdots & \vdots \\
\vdots & \vdots & \ddots & \mathbb{I} & 0 \\
0 & 0 & \cdots & 0 & \mathbb{I}
\end{array}\right] .
$$

The identity (B.9) where matrix polynomials $\mathrm{E}(s)$ and $\mathrm{F}(s)$ have constant determinants can be viewed as the definition of equivalency between matrix polynomial $A(s)$ and its companion polynomial $\mathrm{C}_{A}(s)$.

Let us take a look at the eigenvalue problem for eigenvalue $s$ and eigenvector $\mathrm{x} \in \mathbb{C}^{m \nu}$ associated with companion polynomial $\mathrm{C}_{A}(s)$, that is

$$
(s \mathrm{~B}-\mathrm{A}) \mathrm{x}=0, \quad \mathrm{x}=\left[\begin{array}{c}
x_{0} \\
x_{1} \\
x_{2} \\
\vdots \\
x_{\nu-1}
\end{array}\right] \in \mathbb{C}^{m \nu}, \quad x_{j} \in \mathbb{C}^{m}, \quad 0 \leq j \leq \nu-1,
$$

where

$$
(s \mathrm{~B}-\mathrm{A}) \times=\left[\begin{array}{c}
s x_{0}-x_{1} \\
s x_{1}-x_{2} \\
\vdots \\
s x_{\nu-2}-x_{\nu-1} \\
\sum_{j=0}^{\nu-2} A_{j} x_{j}+\left(s A_{\nu}+A_{\nu-1}\right) x_{\nu-1}
\end{array}\right] .
$$

With equations (B.10) and (B.11) in mind we introduce the following vector polynomial

$$
\mathrm{x}_{s}=\left[\begin{array}{c}
x_{0} \\
s x_{0} \\
\vdots \\
s^{\nu-2} x_{0} \\
s^{\nu-1} x_{0}
\end{array}\right], \quad x_{0} \in \mathbb{C}^{m} .
$$

Not accidentally, the components of the vector $x_{s}$ in its representation (B.12) are in evident relation with the derivatives $\partial_{t}^{j}\left(x_{0} \mathrm{e}^{s t}\right)=s^{j} x_{0} \mathrm{e}^{s t}$. That is just another sign of the intimate relations between the matrix polynomial theory and the theory of systems of ordinary differential equations [Hale, III.4]. 
Theorem 5 (eigenvectors). Let $A(s)$ as in equations (B.1) be regular, that $\operatorname{det}\{A(s)\}$ is not identically zero, and let $m \nu \times m \nu$ matrices $\mathrm{A}$ and $\mathrm{B}$ be defined by equations (B.2). Then the following identities hold

$$
(s \mathrm{~B}-\mathrm{A}) \mathrm{x}_{s}=\left[\begin{array}{c}
0 \\
0 \\
\vdots \\
0 \\
A(s) x_{0}
\end{array}\right], \mathbf{x}_{s}=\left[\begin{array}{c}
x_{0} \\
s x_{0} \\
\vdots \\
s^{\nu-2} x_{0} \\
s^{\nu-1} x_{0}
\end{array}\right]
$$

$$
\operatorname{det}\{A(s)\}=\operatorname{det}\{s \mathrm{~B}-\mathrm{A}\}, \quad \operatorname{det}\{\mathrm{B}\}=\operatorname{det}\left\{A_{\nu}\right\},
$$

where $\operatorname{det}\{A(s)\}=\operatorname{det}\{s \mathrm{~B}-\mathrm{A}\}$ is a polynomial of the degree $m \nu$ if $\operatorname{det}\{\mathrm{B}\}=\operatorname{det}\left\{A_{\nu}\right\} \neq 0$. There is one-to-one correspondence between solutions of equations $A(s) x=0$ and $(s \mathrm{~B}-\mathrm{A}) \mathrm{x}=0$. Namely, a pair $s, \mathrm{x}$ solves eigenvalue problem $(s \mathrm{~B}-\mathrm{A}) \mathrm{x}=0$ if and only if the following equalities hold

$$
\mathbf{x}=\mathbf{x}_{s}=\left[\begin{array}{c}
x_{0} \\
s x_{0} \\
\vdots \\
s^{\nu-2} x_{0} \\
s^{\nu-1} x_{0}
\end{array}\right], \quad A(s) x_{0}=0, \quad x_{0} \neq 0 ; \quad \operatorname{det}\{A(s)\}=0
$$

Proof. Polynomial vector identity (B.13) readily follows from equations (B.11) and (B.12). Identities $(\overline{B .14})$ for the determinants follow straightforwardly from equations $(\overline{B .12}),(\bar{B} .15)$ and $(\overline{B .9})$. If $\operatorname{det}\{\mathrm{B}\}=\operatorname{det}\left\{A_{\nu}\right\} \neq 0$ then the degree of the polynomial $\operatorname{det}\{s \mathrm{~B}-\mathrm{A}\}$ has to be $m \nu$ since $\mathrm{A}$ and $\mathrm{B}$ are $m \nu \times m \nu$ matrices.

Suppose that equations (B.15) hold. Then combining them with proven identity (B.13) we get $(s \mathrm{~B}-\mathrm{A}) \mathrm{x}_{s}=0$ proving that expressions (B.15) define an eigenvalue $s$ and an eigenvector $\mathrm{x}=\mathrm{x}_{s}$.

Suppose now that $(s \mathrm{~B}-\mathrm{A}) \mathbf{x}=0$ where $\mathbf{x} \neq 0$. Combing that with equations (B.11) we obtain

$$
x_{1}=s x_{0}, \quad x_{2}=s x_{1}=s^{2} x_{0}, \cdots, \quad x_{\nu-1}=s^{\nu-1} x_{0},
$$

implying that

$$
\mathbf{x}=\mathbf{x}_{s}=\left[\begin{array}{c}
x_{0} \\
s x_{0} \\
\vdots \\
s^{\nu-2} x_{0} \\
s^{\nu-1} x_{0}
\end{array}\right], \quad x_{0} \neq 0
$$

and

$$
\sum_{j=0}^{\nu-2} A_{j} x_{j}+\left(s A_{\nu}+A_{\nu-1}\right) x_{\nu-1}=A(s) x_{0} .
$$

Using equations (B.17) and identity (B.13) we obtain

$$
0=(s \mathrm{~B}-\mathrm{A}) \mathrm{x}=(s \mathrm{~B}-\mathrm{A}) \mathrm{x}_{s}=\left[\begin{array}{c}
0 \\
0 \\
\vdots \\
0 \\
A(s) x_{0}
\end{array}\right] \text {. }
$$


Equations (B.19) readily imply $A(s) x_{0}=0$ and $\operatorname{det}\{A(s)\}=0$ since $x_{0} \neq 0$. That completes the proof.

Remark 6 (characteristic polynomial degree). Notice that according to Theorem 5 the characteristic polynomial $\operatorname{det}\{A(s)\}$ for $m \times m$ matrix polynomial $A(s)$ has the degree $m \nu$, whereas in linear case $s \mathbb{I}-A_{0}$ for $m \times m$ identity matrix $\mathbb{I}$ and $m \times m$ matrix $A_{0}$ the characteristic polynomial $\operatorname{det}\left\{s \mathbb{I}-A_{0}\right\}$ is of the degree $m$. This can be explained by observing that in the non-linear case of $m \times m$ matrix polynomial $A(s)$ we are dealing effectively with many more $m \times m$ matrices $A$ than just a single matrix $A_{0}$.

Another problem of our particular interest related to the theory of matrix polynomials is eigenvalues and eigenvectors degeneracy and consequently the existence of non-trivial Jordan blocks, that is Jordan blocks of dimensions higher or equal to 2 . The general theory addresses this problem by introducing so-called "Jordan chains" which are intimately related to the theory of system of differential equations expressed as $A\left(\partial_{t}\right) x(t)=0$ and their solutions of the form $x(t)=p(t) e^{s t}$ where $p(t)$ is a vector polynomial, see [GoLaRo, I, II], Baum, 9]. Avoiding the details of Jordan chains developments we simply notice that an important to us point of Theorem 5 is that there is one-to-one correspondence between solutions of equations $A(s) x=0$ and $(s \mathrm{~B}-\mathrm{A}) \mathrm{x}=0$, and it has the following immediate implication.

Corollary 7 (equality of the dimensions of eigenspaces). Under the conditions of Theorem 5 for any eigenvalue $s_{0}$, that is $\operatorname{det}\left\{A\left(s_{0}\right)\right\}=0$, we have

$$
\operatorname{dim}\left\{\operatorname{ker}\left\{s_{0} \mathrm{~B}-\mathrm{A}\right\}\right\}=\operatorname{dim}\left\{\operatorname{ker}\left\{A\left(s_{0}\right)\right\}\right\} .
$$

In other words, the geometric multiplicities of the eigenvalue $s_{0}$ associated with matrices $A\left(s_{0}\right)$ and $s_{0} \mathrm{~B}-\mathrm{A}$ are equal. In view of identity (B.20) the following inequality holds for the (algebraic) multiplicity $m\left(s_{0}\right)$

$$
m\left(s_{0}\right) \geq \operatorname{dim}\left\{\operatorname{ker}\left\{A\left(s_{0}\right)\right\}\right\} .
$$

The next statement shows that if the geometric multiplicity of an eigenvalue is strictly less than its algebraic one than there exist non-trivial Jordan blocks, that is Jordan blocks of dimensions higher or equal to 2 .

Theorem 8 (non-trivial Jordan block). Assuming notations introduced in Theorem 5 let us suppose that the multiplicity $m\left(s_{0}\right)$ of eigenvalue $s_{0}$ satisfies

$$
m\left(s_{0}\right)>\operatorname{dim}\left\{\operatorname{ker}\left\{A\left(s_{0}\right)\right\}\right\} .
$$

Then the Jordan canonical form of companion polynomial $\mathrm{C}_{A}(s)=s \mathrm{~B}-\mathrm{A}$ has a least one nontrivial Jordan block of the dimension exceeding 2.

In particular, if

$$
\operatorname{dim}\left\{\operatorname{ker}\left\{s_{0} \mathrm{~B}-\mathrm{A}\right\}\right\}=\operatorname{dim}\left\{\operatorname{ker}\left\{A\left(s_{0}\right)\right\}\right\}=1,
$$

and $m\left(s_{0}\right) \geq 2$ then the Jordan canonical form of companion polynomial $\mathrm{C}_{A}(s)=s \mathrm{~B}-\mathrm{A}$ has exactly one Jordan block associated with eigenvalue $s_{0}$ and its dimension is $m\left(s_{0}\right)$.

The proof of Theorem 8 follows straightforwardly from the definition of the Jordan canonical form and its basic properties. Notice that if equations (B.23) hold that implies that the eigenvalue 0 is cyclic (nonderogatory) for matrix $A\left(s_{0}\right)$ and eigenvalue $s_{0}$ is cyclic (nonderogatory) for matrix $\mathrm{B}^{-1} \mathrm{~A}$ provided $\mathrm{B}^{-1}$ exists. We remind that an eigenvalue is called cyclic (nonderogatory) if its geometric multiplicity is 1. A square matrix is called cyclic (nonderogatory) if all its eigenvalues are cyclic [BernM, 5.5]. 


\section{Appendix C. Notations}

- $\mathbb{C}$ is a set of complex number.

- $\bar{s}$ is complex-conjugate to complex number $s$

- $\mathbb{C}^{n}$ is a set of $n$ dimensional column vectors with complex complex-valued entries.

- $\mathbb{C}^{n \times m}$ is a set of $n \times m$ matrices with complex-valued entries.

- $\mathbb{R}^{n \times m}$ is a set of $n \times m$ matrices with real-valued entries.

- $\operatorname{dim}(W)$ is the dimension of the vector space $W$.

- $\operatorname{ker}(A)$ is the kernel of matrix $A$, that is the vector space of vector $x$ such that $A x=0$.

- $\operatorname{det}\{A\}$ is the determinant of matrix $A$.

- $\chi_{A}(s)=\operatorname{det}\left\{s \mathbb{I}_{\nu}-A\right\}$ is the characteristic polynomial of a $\nu \times \nu$ matrix $A$.

- $\mathbb{I}_{\nu}$ is $\nu \times \nu$ identity matrix.

- $M^{\mathrm{T}}$ is a matrix transposed to matrix $M$.

- EL stands for the Euler-Lagrange (equations).

Data Availability: The data that supports the findings of this study are available within the article.

Acknowledgment: This research was supported by AFOSR grant \# FA9550-19-1-0103.

\section{REFERENCES}

[ArfWeb] Arfken G. and Weber H., Mathematical Methods for Physicists - A Comprehensive Guide, 7th edn., Academic Press, 2013. A

[Nusi] Barker R., Booske J., Luhmann N. and G. Nusinovich, Modern Microwave and Millimeter-Wave Power Electronics, Wiley, 2005. 1, 1

[Baum] Baumgartel H., Analytic Perturbation Theory for Matrices and Operators, Birkhauser, 1985. B, B

[BernM] Bernstein D., Matrix Mathematics: Theory, Facts, and Formulas, 2 edn., Princeton University Press, 2009. B

[BoosVE] Booske J. et.al., Vacuum Electronic High Power Terahertz Source, (traveling wave tubes, TWT), IEEE Trans. on Terahertz Sci. and Tech., 1, No. 1, pp. 54-78, (2011). 1

[Burt] Burtsev A. et. al., Features of the Development of Electron-Optical Systems for Pulsed Terahertz Traveling-Wave Tubes, Tech. Phys., 63, pp. 452-459, (2018). 1

[CheN] Chen W. et. al., Exceptional points enhance sensing in an optical microcavity, Nature, 548, 192-196, (2017). 1

[FigTWTbk] Figotin A., An Analytic Theory of Multi-stream Electron Beams in Traveling Wave Tubes, World Scientific, 2020. 1, 1, 2, 2, 2, 2.1, 2.1, 2.1, 2.2, 2.2, 2.3, 2.4, 2.4, 3.1

[FigSynbJ] Figotin A., Synthesis of lossless electric circuits based on prescribed Jordan forms, J. Math. Phys., 61, 122703, (2020). 1

[FigPert] Figotin A., Perturbations of circuit evolution matrices with Jordan blocks, arXiv:2011.09425 [physics.class-ph], 2020. 1, 4, 4.2

[FigRey1] Figotin A. and Reyes G., Multi-transmission-line-beam interactive system, J. Math. Phys., 54, 111901, (2013). 1

[Foll] Folland G., Fourier analysis and its applications, Wadsworth \& Brooks, 1992. A, A

[Gilm1] Gilmour A., Principles of Klystrons, Traveling Wave Tubes, Magnetrons, Cross-Field Amplifiers, and Gyrotrons, Artech House, 2011.1, 1.

[Gilm] Gilmour A., Principles of Traveling Wave Tubes, Artech House, 1994. 1

[GoLaRo] Gohberg I., Lancaster P., L. and Rodman L., Matrix Polynomials, SIAM, 2009.. B, B

[Hale]

[Kato]

Hale J., Ordinary Differential Equations, 2nd ed., Krieger Publishing Co., 1980. B

[KNAC] Kazemi H., Nada M., Mealy T., Abdelshafy A. and Capolino F., Exceptional Points of Degeneracy Induced by Linear Time-Periodic Variation, Phys. Rev. Applied, 11, 014007 (2019). 1

[MAEAD] Minenna D., Andre F., Elskens Y., Auboin J-F., Doveil F., The Traveling-Wave Tube in the History of Telecommunication, Eur. Phys. J., 44(1), 1-36, 2019.1 
[OGC] Othman M., Galdi V. and Capolino F., Exceptional points of degeneracy and PT symmetry in photonic coupled chains of scatterers, Phys. Rev. B, 95, 104305 (2017). 1

[OTC] Othman M., Tamma V., and Capolino F., Theory and new amplification regime in periodic multimodal slow wave structures with degeneracy interacting with an electron beam, IEEE Trans. Plasma Sci., 44, 594 (2016). 1

[OVFC] Othman M, Veysi M., A. Figotin A. and Capolino F., Low starting electron beam current in degenerate band edge oscillators, IEEE Trans. Plasma Sci., 44, 918 (2016). 1

[OVFC1] Othman M., Veysi M., Figotin A. and Capolino F., Giant amplification in degenerate band edge slow-wave structures interacting with an electron beam, Phys. Plasmas, 23, 033112 (2016). [1

[PeLiXu] Peng C., Li Z., and Xu A., Rotation sensing based on a slow-light resonating structure with high group dispersion, Appl. Opt., 46, 4125 (2007). 1

[PierTWT] Pierce J., Traveling-Wave Tubes, D. van Nostrand, 1950. 1. 2

[Pier51] Pierce J., Waves in Electron Streams and Circuits, Bell Sys. Tech. J., 30, 626-651, 1951. 1, 2

[SchaB] Schachter L., Beam-Wave Interaction in Periodic and Quasi-Periodic Structures, 2nd edn., Springer, 2011. 10 1

[Tsim] Tsimring S., Electron Beams and Microwave Vacuum Electronics, Wiley, 2007.1, 1,

[vdWae1] van der Waerden B., Algebra, vol. 1, Springer, 2003.

[VPFC] Veysi M., Othman M., Figotin A. and Capolino F., Degenerate band edge laser, Phys. Rev. B, 97, 195107 (2018). 1

[Wie] Wiersig J., Enhancing the Sensitivity of Frequency and Energy Splitting Detection by Using Exceptional Points - Application to Microcavity Sensors for Single-Particle Detection, Phys. Rev. Lett., 112, 203901 (2014). 1

[Wie1] Wiersig J., Sensors operating at exceptional points: General theory, Phys. Rev. A, 93, 033809 (2016). 1

University of California at Irvine, CA 92967 